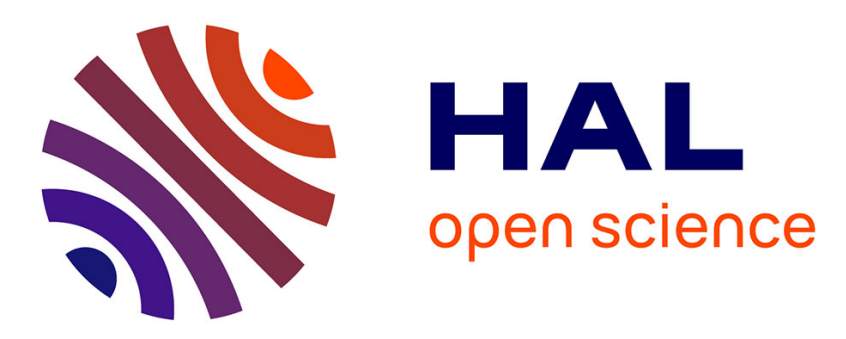

\title{
Robust calibration of numerical models based on relative regret
}

Victor Trappler, Élise Arnaud, Arthur Vidard, Laurent Debreu

\section{To cite this version:}

Victor Trappler, Élise Arnaud, Arthur Vidard, Laurent Debreu. Robust calibration of numerical models based on relative regret. Journal of Computational Physics, 2021, 426, pp.109952:1-19. 10.1016/j.jcp.2020.109952 . hal-02464780v3

\section{HAL Id: hal-02464780 \\ https://hal.science/hal-02464780v3}

Submitted on 6 Nov 2020

HAL is a multi-disciplinary open access archive for the deposit and dissemination of scientific research documents, whether they are published or not. The documents may come from teaching and research institutions in France or abroad, or from public or private research centers.
L'archive ouverte pluridisciplinaire HAL, est destinée au dépôt et à la diffusion de documents scientifiques de niveau recherche, publiés ou non, émanant des établissements d'enseignement et de recherche français ou étrangers, des laboratoires publics ou privés. 


\title{
Robust calibration of numerical models based on relative regret
}

\author{
Victor Trappler ${ }^{\mathrm{a}, *}$, Élise Arnaud ${ }^{\mathrm{a}}$, Arthur Vidard ${ }^{\mathrm{a}}$, Laurent Debreu ${ }^{\mathrm{a}}$ \\ ${ }^{a}$ Univ. Grenoble Alpes, Inria, CNRS, Grenoble INP ${ }^{1}$, LJK, 38000 Grenoble, France
}

\begin{abstract}
Classical methods of calibration usually imply the minimisation of an objective function with respect to some control parameters. This function measures the error between some observations and the results obtained by a numerical model. In the presence of uncontrollable additional parameters that we model as random inputs, the objective function becomes a random variable, and notions of robustness have to be introduced for such an optimisation problem.

In this paper, we are going to present how to take into account those uncertainties by defining the relative-regret. This quantity allow us to compare the value of the objective function to its best performance achievable given a realisation of the random additional parameters. By controlling this relative-regret using a probabilistic constraint, we can then define a new family of estimators, whose robustness with respect to the random inputs can be adjusted.
\end{abstract}

Keywords: Calibration, Robust optimisation, Relative-regret, Shallow-water equations

\section{Introduction}

Numerical models are widely used to study or forecast natural phenomena and improve industrial processes. However, by essence models only partially represent reality and sources of uncertainties are ubiquitous (discretisation errors, missing physical processes, poorly known boundary conditions). Moreover, such uncertainties may be of different nature. [1] proposes to consider two categories of uncertainties. On the one

\footnotetext{
* Corresponding author

Email address: victor.trappler@univ-grenoble-alpes.fr (Victor Trappler)

${ }^{1}$ Institute of Engineering Univ. Grenoble Alpes

Preprint submitted to Journal of Computational Physics
}

October 29, 2020 
hand aleatoric uncertainties, coming from the inherent variability of a phenomenon, e.g. intrinsic randomness of some environmental variables. On the other hand, epistemic uncertainties coming from a lack of knowledge about the properties and conditions of the phenomenon underlying the behaviour of the system under study. The latter can be accounted for through the introduction of ad-hoc correcting terms in the numerical model, that need to be properly estimated. Thus, reducing the epistemic uncertainty can be done through parameters estimation approaches. This is usually done using optimal control techniques, leading to an optimisation of a well chosen cost function which is typically built as a comparison with reference observations. An application of such an approach, in the context of ocean circulation modelling, is the estimation of ocean bottom friction parameters, as done in $[2,3,4]$. Moreover, as such studies are often performed at a coastal and regional scale, those models are often designed with open boundary conditions, and require external forcings, such as tidal and wind forcing. Those are then a source of aleatoric uncertainties, that should be taken into account as in [5].

If we overlook the aleatoric uncertainties by choosing a specific outcome, the optimal control of the parameters to be estimated can lead to localized optimisation [6] and overcalibration, that is choosing a value that is optimal for the given situation. This value does not carry the optimality to other situations. In geophysics and especially in hydrological models, this overcalibration may lead to the appearance of abberations in the predictions as those uncertainties become prevalent sources of errors. In hydrology, uncertainties are the principal culprit of the existence of so called "Hydrological monsters" [7], that are calibrated models that perform really badly because some uncertainties in the modelling have been omitted, such as measurements errors. In flood modelling [8], the aleatoric uncertainty come from the structure of the model, and neglecting those uncertainties leads to underestimating hazard. In other domains as well, the aleatoric uncertainties can represent some manufacturing errors or environmental conditions such as wind direction and speed in wind turbine modelling [9] or atmospheric conditions in aerospace vehicle design [10], thus they represent an important role in risk management.

It is then crucial to be able to take into account aleatoric uncertainties in optimisation problems. This consideration is called robust optimisation, or also robust design in [11], or optimisation under uncertainties [12, 13, 14]. Furthermore, the distinction is 
sometimes made between stochastic optimisation and robust optimisation, depending on the knowledge available on the aleatoric uncertainties.

Let us denote $\mathbf{k} \in \mathbb{K}$ the parameter to be estimated in order to reduce epistemic uncertainties. We assume that the aleatoric uncertainties can be modelled as a random vector $\mathbf{U}$ whose sample space is $\mathbb{U}$. The probability measure associated with $\mathbf{U}$ is $\mathbb{P}_{\mathbf{U}}$, and its density, if it exists, is $p_{\mathbf{U}}$. This distribution is assumed to be known, and that it is possible to sample from it. This choice is motivated by the fact that in various applications, the aleatoric uncertainties come from expert knowledge, empirical observations, or the knowledge acquired using other models, for instance by ensemble assimilation. However, if it is not the case, [15] provides a comprehensive review of optimisation under uncertainty, especially on the modelling and sampling of the aleatoric uncertainties. Since the source of the aleatoric uncertainties is considered external, the choice of $\mathbf{k}$ does not have any influence on the distribution of the random variable $\mathbf{U}$, and therefore the aleatoric and epistemic uncertainties are assumed independent.

The cost function $J(\mathbf{k}, \mathbf{U})$ is a random variable in this context. It is most often defined as the squared norm of a given function $\mathcal{G}(\mathbf{k}, \mathbf{U})$

$$
J(\mathbf{k}, \mathbf{U})=\frac{1}{2}\|\mathcal{G}(\mathbf{k}, \mathbf{U})\|^{2}
$$

For instance, in data assimilation, $J$ describes a distance between the output of the numerical model and given observed data, plus generally some regularization terms. An example of such a function will be treated Section 4 .

For a practical purpose, we assume that for every realisation $\mathbf{u} \in \mathbb{U}$ of $\mathbf{U}$, finding $\mathbf{k}_{\mathbf{u}} \in \mathbb{K}$ that minimises the cost function $\mathbf{k} \mapsto J(\mathbf{k}, \mathbf{U}=\mathbf{u})$ is a well-posed problem, and that the optimum is unique. Additionally, the following assumptions are made: the cost function is strictly positive, and $\forall \mathbf{k} \in \mathbb{K}$, the random variable $J(\mathbf{k}, \mathbf{U})$ has finite firstand second-order moments.

In this paper, we aim at finding $\hat{\mathbf{k}}$ a robust estimator of $\mathbf{k}$. The definition of robustness differs depending on the context in which it is used. Indeed, one definition of the robustness of an estimate is a measure of the sensibility of said estimate to outliers [16]. This leads to the introduction of robust norms in data assimilation [17]. In a Bayesian framework, robustness may refer to the sensitivity to a wrong specification of the priors [18]. 
As the distribution of $\mathbf{U}$ is assumed known, we are not going to consider distributional robustness, which deals with uncertainties on the distribution of $\mathbf{U}$ [19].

Throughout this paper, robust has to be understood as satisfactory for a broad range of $\mathbf{u}$, and/or as insensitive as possible to uncertainties encompassed in $\mathbf{U}$.

The usual practice consists in neglecting the variability of $\mathbf{U}$ by setting it to an $a$ priori value $\mathbf{u}^{b}$. In this case, $\hat{\mathbf{k}}$ is set to the optimum $\mathbf{k}_{\mathbf{u}^{b}}$ of $J\left(\mathbf{k}, \mathbf{U}=\mathbf{u}^{b}\right)$. There is no guarantee on the performance of $\hat{\mathbf{k}}$ if the calibrated model is used for predictions, as the estimated value will compensate the error made by a possibly wrong specification of $\mathbf{u}^{b}$ : this may be a case of overcalibration In a data assimilation context, this situation appears if $\mathbf{u}^{b}$ does not properly represent the conditions in which the observations have been obtained.

Another strategy, that consists in minimising $J$ over the joint space $\mathbb{K} \times \mathbb{U}$, is not always possible or relevant. Indeed, joint optimisation increases the complexity of the optimisation, and the computed estimation of $\hat{\mathbf{k}}$ has no reason to be robust in the end: this kind of method does not take into consideration the intrinsic variability of the environmental variable. The worst-case approach [20] is another popular method, and is based on the minimisation with respect to $\mathbf{k}$ of the maximum of the cost function for $\mathbf{u} \in \mathbb{U}: \min _{\mathbf{k}} \max _{\mathbf{u}} J(\mathbf{k}, \mathbf{U}=\mathbf{u})$. This approach may yield over-conservative solutions, and does not take into account the random nature of $\mathbf{U}$.

Accounting for the probabilistic nature of $\mathbf{U}$ leads to study the distribution of the random variable $J(\mathbf{k}, \mathbf{U})$, or the distribution of its minimisers $\mathbf{k}_{\mathbf{U}}$. The latter is referred as the distribution of the conditional minimisers, notion that appeared notably in [21] and in [22] for a global optimisation purpose. Both approaches and related robust estimates are described in Section 2. Section 3 introduces a new class of estimators, by relaxing the constraint of optimality and defining regions of acceptability, similarly as [23] in discrete combinatorial problems, or $[24,25]$ in operations research. The rationale behind this relaxation is to be able to construct an estimate $\hat{\mathbf{k}}$ which produces values of the cost function close enough from the minimal cost attainable given the configuration induced by $\mathbf{u} \in \mathbb{U}$, with high enough probability. This similarity will be measured using the relative regret. By adjusting either the relaxation or the confidence level, we can then define the RRE, the relative-regret family estimators. Illustration of the various described 
methods are given on a numerical example in Section 4.

\section{Classical robust estimators}

As mentioned before, robustness can be understood as satisfactory for a broad range of $\mathbf{u}$, and/or as insensitive as possible to uncertainties encompassed in $\mathbf{U}$. Under this definition, one may design robust estimators of $\mathbf{k}$, either by using the moments of the cost function; or by exploiting the distribution of its minimisers.

\subsection{Optimisation of the moments}

Let us define $\mu(\mathbf{k})$ and $\sigma^{2}(\mathbf{k})$, the expected value and the variance of the cost variable for a given $\mathbf{k}$ as

$$
\begin{aligned}
\mu(\mathbf{k}) & =\mathbb{E}_{\mathbf{U}}[J(\mathbf{k}, \mathbf{U})]=\int_{\mathbb{U}} J(\mathbf{k}, \mathbf{u}) p_{\mathbf{U}}(\mathbf{u}) \mathrm{d} \mathbf{u} \\
\sigma^{2}(\mathbf{k}) & =\operatorname{Var}_{\mathbf{U}}[J(\mathbf{k}, \mathbf{U})]=\int_{\mathbb{U}}(J(\mathbf{k}, \mathbf{u})-\mu(\mathbf{k}))^{2} p_{\mathbf{U}}(\mathbf{u}) \mathrm{d} \mathbf{u}
\end{aligned}
$$

Minimising the expectation leads to the estimate $\mathbf{k}_{\mathbb{E}}$ defined by:

$$
\mathbf{k}_{\mathbb{E}}=\underset{\mathbf{k} \in \mathbb{K}}{\arg \min } \mu(\mathbf{k})
$$

In order to take into account the spread around the mean value, one can choose to minimise the variance, leading to $\mathbf{k}_{\mathbb{V}}$ :

$$
\mathbf{k}_{\mathbb{V}}=\underset{\mathbf{k} \in \mathbb{K}}{\arg \min } \sigma^{2}(\mathbf{k})
$$

A lot of different methods are readily available to solve these minimisation problems. For instance, stochastic Sample Approximation $[26,27]$ is based on a finite and fixed set of samples $\left\{\mathbf{u}^{i}\right\}_{i=1 \ldots N}$ of $\mathbf{U}$. The estimations at a given $\mathbf{k}$ are computed using standard Monte Carlo, resulting in the following optimisation problems:

$$
\hat{\mathbf{k}}_{\mathbb{E}}=\underset{\mathbf{k} \in \mathbb{K}}{\arg \min } \sum_{i=1}^{N} J\left(\mathbf{k}, \mathbf{u}^{i}\right)
$$

and

$$
\hat{\mathbf{k}}_{\mathbb{V}}=\underset{\mathbf{k} \in \mathbb{K}}{\arg \min } \sum_{i=1}^{N}\left(\begin{array}{c}
\left.J\left(\mathbf{k}, \mathbf{u}^{i}\right)-\sum_{j=1}^{N} J\left(\mathbf{k}, \mathbf{u}^{j}\right)\right)^{2} \\
5
\end{array}\right.
$$


If computationally affordable, one can perform these estimations on a regular grid on $\mathbb{K} \times \mathbb{U}$. In case of expensive computer code, one can build a meta model to ease the minimisation, such as Gaussian processes [28]. Even though $\mathbf{k}_{\mathbb{E}}$ is a reasonable choice, there is no guarantee that $J\left(\mathbf{k}_{\mathbb{E}}, \mathbf{U}=\mathbf{u}\right)$ will not reach catastrophic level for some $\mathbf{u}$. On the other hand, using $\mathbf{k}_{\mathbb{V}}$ will ensure stability of the cost function, but without any control of its performance. Ideally, one would want to have a small expectation and a small variance at the same time. Multi-objective optimisation is a proper tool to deal with these simultaneous and sometimes concurrent objectives, for exemple by computing the Pareto front of $\left(\mu(\mathbf{k}), \sigma^{2}(\mathbf{k})\right)$ as done in [29].

As the computation of this Pareto front is usually hard and expensive, alternative strategies based on the minimisation of a scalarized version of the vector of objectives are often considered. Some are based on a weighted sum of the objectives, as presented in [30] and in [31], while some others are based on the minimisation of one of the objectives under constraints on the others, as performed in [32]. Both of these methods are based on an delicate choice of weights or of constraints before any computation. This choice relies heavily on a knowledge of the properties of the cost function.

To summarise, even though the notions of mean and variance are quite easily understood, getting a satisfactory estimator is not that straightforward. One could instead consider how often a particular value $\mathbf{k}$ is a minimiser of the cost function, leading to the notion of most probable estimate, as explained in the next subsection.

\subsection{Most probable estimate}

Let us consider the minimal cost attainable in each configuration brought by $\mathbf{u}$. The resulting conditional minimum is denoted $J^{*}$ :

$$
J^{*}: \mathbf{u} \in \mathbb{U} \longmapsto J^{*}(\mathbf{u})=\min _{\mathbf{k} \in \mathbb{K}} J(\mathbf{k}, \mathbf{U}=\mathbf{u})
$$

Similarly, the function of conditional minimisers can then defined by:

$$
\mathbf{k}^{*}: \mathbf{u} \in \mathbb{U} \longmapsto \mathbf{k}^{*}(\mathbf{u})=\mathbf{k}_{\mathbf{u}}=\underset{\mathbf{k} \in \mathbb{K}}{\arg \min } J(\mathbf{k}, \mathbf{U}=\mathbf{u})
$$

Using this function, we can define the corresponding random variable $\mathbf{K}^{*}$ as

$$
\begin{aligned}
& \mathbf{K}^{*}=\mathbf{k}^{*}(\mathbf{U}), \\
& 6
\end{aligned}
$$


and its associated density function $p_{\mathbf{K}^{*}}(\mathbf{k})$, that will be further referred as the density of minimisers. The mode of this density is called the Most Probable Estimate (MPE) and is noted $\mathbf{k}_{\mathrm{MPE}}$ :

$$
\mathbf{k}_{\mathrm{MPE}}=\underset{\mathbf{k} \in \mathbb{K}}{\arg \max } p_{\mathbf{K}^{*}}(\mathbf{k})
$$

To give some intuition on this estimate, let us imagine that the distribution of minimisers is a dirac centered on $\mathbf{k}_{\mathrm{MPE}}$. Then it would mean that this estimate is the minimiser of the cost function whatever the realisation of the uncertain variable, therefore optimal in all conditions. If the distribution $p_{\mathbf{K}^{*}}$ is heavily dominated by a single value, the MPE may be a good candidate for robust control. This is not so obvious in the case of a multimodal distribution.

In general, an analytical form of $p_{\mathbf{K}^{*}}$ is impossible to obtain, so an estimation $\hat{p}_{\mathbf{K}^{*}}$ must be used, and its maximum computed to get the MPE. In the rest of the paper, the hat notation will indicate an estimation using numerical values of the underlying theoretical quantity. Once again, a set of samples $\left\{\mathbf{u}^{i}\right\}_{i=1 \ldots N}$ can be used to compute the set $\left\{\mathbf{k}_{\mathbf{u}^{i}}\right\}_{i=1 \ldots N}$, from which one can approximate $p_{\mathbf{K}^{*}}$. The resulting approximation and therefore its mode, is sensitive to the density estimation method. Main methods are KDE (Kernel Density Estimation) [33], and EM (Expectation-Maximisation) [34].

$\mathrm{KDE}$ is a non-parametric estimation technique based on the use of a kernel function $f$. Assuming an isotropic kernel, the estimation has the following form:

$$
\hat{p}_{\mathbf{K}^{*}}(\mathbf{k})=\frac{1}{N h^{\operatorname{dim} \mathbb{K}}} \sum_{i=1}^{N} f\left(\frac{\mathbf{k}-\mathbf{k}_{\mathbf{u}^{i}}}{h}\right)
$$

where $h$ is the bandwidth. In a multidimensional setting, one usually consider a kernel based on the product of 1D kernels, applied independently to all components: $f(\mathbf{k})=\prod_{j=1}^{\operatorname{dim} \mathbb{K}} f_{1 \mathrm{D}}\left(k^{(j)}\right)$ where $k^{(j)}$ is the $j$-th component of $\mathbf{k}$. There is wide choice of available $f_{1 \mathrm{D}}$, and a popular choice is the Gaussian kernel $f_{1 \mathrm{D}}(x)=\frac{1}{\sqrt{2 \pi}} \exp \left(-x^{2} / 2\right)$.

The EM algorithm can also be used to estimate the density, by minimising the statistical distance between the empirical distribution and a mixture of $\nu$ Gaussian densities. The estimation has then the following form:

$$
\hat{p}_{\mathbf{K}^{*}}(\mathbf{k})=\sum_{i=1}^{\nu} \pi_{i} \phi\left(\mathbf{k} ; \mathbf{m}_{i}, \boldsymbol{\Sigma}_{i}\right)
$$


where $\phi(\cdot ; \mathbf{m}, \mathbf{\Sigma})$ is the probability density function of the normal distribution of mean $\mathbf{m}$ and covariance matrix $\boldsymbol{\Sigma}$, and $\left\{\pi_{i}\right\}_{i=1 \ldots \nu}$ are the mixing coefficients.

In practice, despite the fact that those methods are well established, using them in a plug-in approach has some flaws. One of the basic assumption of density estimation is to assume that $\mathbf{K}^{*}$ is a continuous random variable, hypothesis that may be violated. Worse, the notion of mode is not well defined when the distribution of the minimisers is a discrete-continuous mixture. This may result in inconsistent estimations of $\hat{\mathbf{k}}_{\mathrm{MPE}}$ when using different methods as illustrated in next subsection.

\subsection{Numerical illustration}

Before going further in the explanation of our approach, let us illustrate the nature of previously detailed estimators, $\hat{\mathbf{k}}_{\mathbb{E}}, \hat{\mathbf{k}}_{\mathbb{V}}$ and $\hat{\mathbf{k}}_{\mathrm{MPE}}$ on two analytical cost functions. These functions are based on the Branin-Hoo's function, slightly modified to ensure strict positivity:

$$
\begin{gathered}
\mathrm{BH}\left(x_{1}, x_{2}\right)=\frac{1}{51.95}\left[\left(\bar{x}_{2}-\frac{5.1 \bar{x}_{1}^{2}}{4 \pi^{2}}+\frac{5 \bar{x}_{1}}{\pi}-6\right)^{2}+\left(10-\frac{10}{8 \pi}\right) \cos \left(\bar{x}_{1}\right)-44.81\right]+2 \\
\text { with } \bar{x}_{1}=3 x_{1}-5, \quad \bar{x}_{2}=3 x_{2}
\end{gathered}
$$

Using Eq. (14), we define the two cost functions on $\mathbb{K} \times \mathbb{U}=[0,5] \times[0,5]$ as:

$$
\begin{array}{r}
J_{\mathrm{BH}}:(\mathbf{k}, \mathbf{u}) \mapsto \mathrm{BH}(\mathbf{k}, \mathbf{u}) \\
J_{\text {BHswap }}:(\mathbf{k}, \mathbf{u}) \mapsto \mathrm{BH}(\mathbf{u}, \mathbf{k})
\end{array}
$$

Even though the functions are quite similar, the asymmetric roles of $\mathbf{k}$ and $\mathbf{u}$ cause different behaviour in the estimations.

The random variable $\mathbf{U}$ is assumed to be uniformly distributed over $\mathbb{U}$. The estimations are based on a $1000 \times 1000$ regular grid over $\mathbb{K} \times \mathbb{U}$. Both cost functions are shown on the top of Figure 1.

The left (resp. right) column stands for $J_{\mathrm{BH}}$ (resp. $J_{\mathrm{BHswap}}$ ). Functions $\mu(\mathbf{k})$ and $\sigma(\mathbf{k})$ are drawn on the bottom row, respectively in purple and green. The corresponding minimisers $\hat{\mathbf{k}}_{\mathbb{E}}$ and $\hat{\mathbf{k}}_{\mathbb{V}}$ are also plotted. On this figure, we can observe that $\hat{\mathbf{k}}_{\mathbb{E}}$ and $\hat{\mathbf{k}}_{\mathbb{V}}$ are close for $J_{\mathrm{BH}}$, while being significantly different for $J_{\mathrm{BH} \text { swap }}$. 

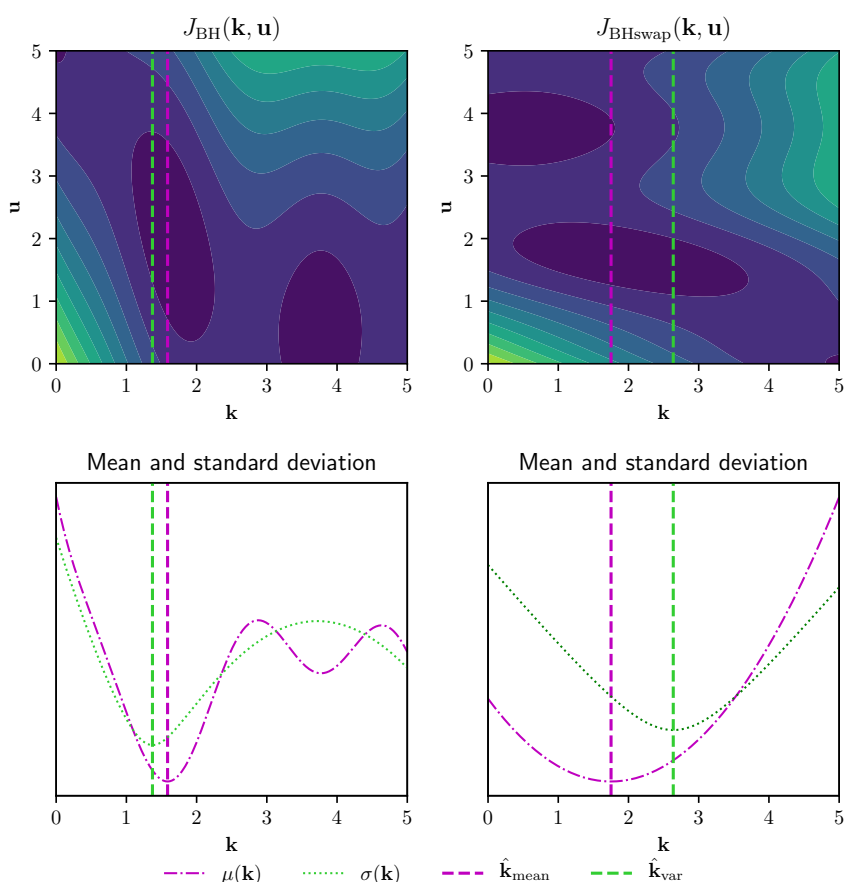

Figure 1: The left column concerns $J_{\mathrm{BH}}$, while the right one deals with $J_{\mathrm{BH} \text { swap }}$. Contours of both functions are plotted on the top, and curves of $\mu(\mathbf{k})$ and $\sigma(\mathbf{k})$ are shown on the bottom (respective scales are not displayed). Estimates $\hat{\mathbf{k}}_{\mathbb{E}}$ and $\hat{\mathbf{k}}_{\mathbb{V}}$ are plotted with the dashed line.

Similarly, estimations of $\hat{\mathbf{k}}_{\mathrm{MPE}}$ are depicted on Figure 2. The top row shows the contours of both functions as well as the set of conditional minimisers $\left\{\mathbf{k}_{\mathbf{u}^{i}}\right\}_{1 \leq i \leq N}$ in red, as defined in Eq. (9). The bottom row presents three approximations of the density of minimisers: the histogram in grey (bin size selected using Freedman-Diaconis from [35]), the result of a kernel density estimation (KDE) with Gaussian kernels in red (using Scott's rule from [36] for bandwidth selection), and the estimation by a Gaussian mixture, calculated with the EM algorithm. The number of Gaussians has been fixed to 3, a guess based on the general shape of the histogram. Respective estimations of $\hat{\mathbf{k}}_{\mathrm{MPE}}$ are also depicted using dashed lines.

For $J_{\mathrm{BHswap}}$, we can observe that those three methods give consistent results, as $\hat{\mathbf{k}}_{\mathrm{MPE}, \mathrm{KDE}}=\hat{\mathbf{k}}_{\mathrm{MPE}, \mathrm{EM}}=\hat{\mathbf{k}}_{\mathrm{MPE}, \text { histogram }} \approx 0.8$. This is not the case for $J_{\mathrm{BH}}$ : using Kernel density estimation (Gaussian), the estimation of $\mathbf{k}_{\mathrm{MPE}}$ is $\hat{\mathbf{k}}_{\mathrm{MPE}, \mathrm{KDE}} \approx 1.5$, while using the histogram and Gaussian mixture, $\hat{\mathbf{k}}_{\mathrm{MPE}, \text { histogram }}=\hat{\mathbf{k}}_{\mathrm{MPE}, \mathrm{EM}}=3.8$. 

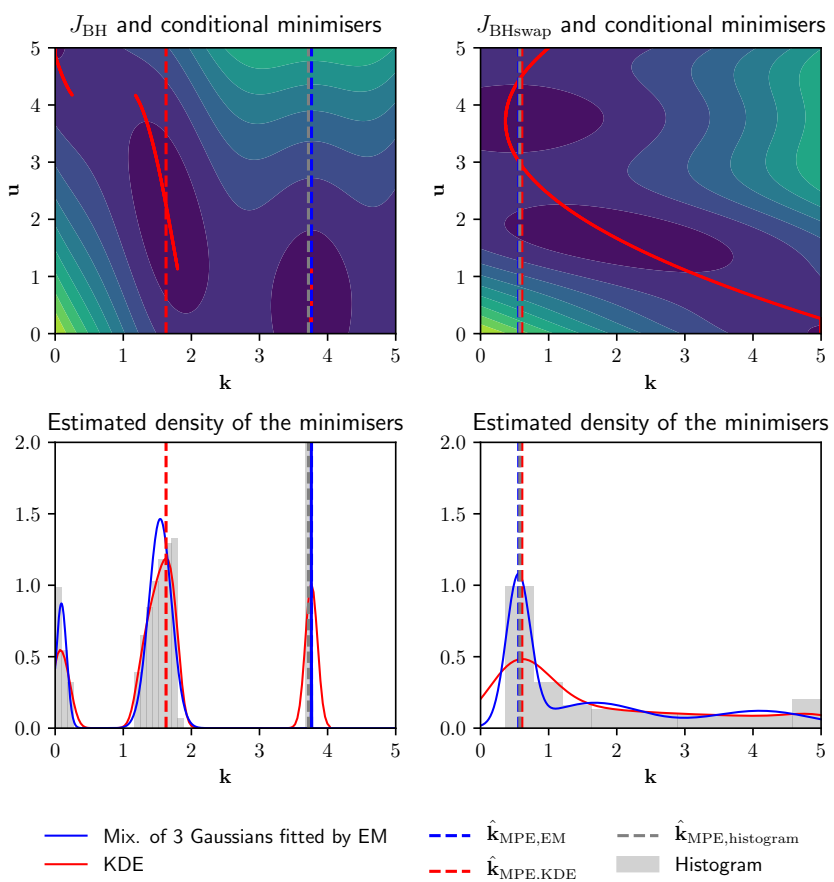

Figure 2: Top Left: $J_{\mathrm{BH}}$ along with conditional minimisers in red. Bottom left: Estimated densities using KDE and EM algorithm and the histogram. The dashed lines indicate the MPE found using those methods. Right: Same quantities with $J_{\text {BHswap }}$.

This difference is explained by the accumulation of minimisers at this point: this challenges the assumption that $\mathbf{K}^{*}$ is continuous. As the density estimation techniques traditionally assume this continuity, the EM algorithm fits this using a normal distribution with a very small variance, while the KDE considers a sum of Gaussian kernels of constant bandwidth, located at the same point. This particular problem highlights an issue with $\mathbf{k}_{\mathrm{MPE}}$, as its estimation is possibly sensitive to the density approximation procedure.

Instead of just considering the optimal minimisers, we introduce a bit of leeway, and look for "acceptably not optimal" parameters. This slackness takes the form of a relaxation coefficient and its choice defines a new family of robust estimators, where each one of its member carries information on its robustness through this coefficient. 


\section{Relative regret-based family of estimators}

\subsection{Relaxing the optimality constraint}

The density of minimisers has been estimated by optimising $\mathbf{k} \mapsto J(\mathbf{k}, \mathbf{U}=\mathbf{u})$ over $\mathbb{K}$ for different realisations of $\mathbf{u}$. Instead of focusing on optimal values, we propose to construct an acceptable neighbourhood in terms of performances of the cost function as well. In order to do so, we are going to introduce a relaxation coefficient $\alpha \geq 1$, and given $\mathbf{u} \in \mathbb{U}$, we say that $\mathbf{k} \in \mathbb{K}$ is acceptable when $J(\mathbf{k}, \mathbf{U}=\mathbf{u}) \leq \alpha J^{*}(\mathbf{u})$. Using the strict positivity of the objective function, we can define the relative-regret as the ratio $J(\mathbf{k}, \mathbf{U}=\mathbf{u}) / J^{*}(\mathbf{u})$, and the acceptability of $\mathbf{k}$ will then be tied to the maximal value taken by this ratio.

In this context, for a given $\mathbf{k}$, the set $R_{\alpha}(\mathbf{k}) \subseteq \mathbb{U}$ is defined as the set of $\mathbf{u}$, for which $\mathbf{k}$ is acceptable:

$$
R_{\alpha}(\mathbf{k})=\left\{\mathbf{u} \in \mathbb{U} \mid J(\mathbf{k}, \mathbf{u}) \leq \alpha J^{*}(\mathbf{u})\right\}
$$

Figure 3 details the successive steps for the construction of the set $R_{\alpha}$. First, the conditional minimisers are computed, as shown on the top plots. Afterwards, for a given level $\alpha=1.5$, the set of acceptable $\mathbf{k}$ can be identified for each $\mathbf{u} \in \mathbb{U}$, as shown on the bottom left plot. Finally, the region $R_{\alpha}(\mathbf{k})$ is the subset of $\mathbb{U}$ for which $\mathbf{k}$ is acceptable, as represented with a vertical slice on the bottom right plot.

Introducing the random nature of $\mathbf{U}$, one can define $\Gamma_{\alpha}(\mathbf{k})$ as the probability that $\mathbf{k}$ is acceptable given $\alpha$ :

$$
\Gamma_{\alpha}(\mathbf{k})=\mathbb{P}_{\mathbf{U}}\left[\mathbf{U} \in R_{\alpha}(\mathbf{k})\right]=\mathbb{P}_{\mathbf{U}}\left[J(\mathbf{k}, \mathbf{U}) \leq \alpha J^{*}(\mathbf{U})\right]
$$

In other words, $\Gamma_{\alpha}(\mathbf{k})$ is the probability that $J(\mathbf{k}, \mathbf{U})$ is between $J^{*}(\mathbf{U})$ and $\alpha J^{*}(\mathbf{U})$.

Noting that without relaxation, i.e. when $\alpha$ is set to $1, \Gamma_{1}$ is non-zero if the set $\left\{\mathbf{u} \in \mathbb{U} \mid J(\mathbf{k}, \mathbf{U}=\mathbf{u})=J^{*}(\mathbf{u})\right\}$ has non-zero measure with respect to $\mathbb{P}_{\mathbf{U}}$. It happens when the distribution of $\mathbf{K}^{*}$ presents atoms.

This linked to the definition of the distribution of the minimisers $\mathbf{K}^{*}$. For instance, if $\mathbb{K}$ is a discrete set, $\mathbf{K}^{*}$ is a discrete random variable, and we can rewrite $\Gamma_{1}$ as $\Gamma_{1}(\mathbf{k})=$ $\mathbb{P}_{\mathbf{U}}\left[J(\mathbf{k}, \mathbf{U})=J^{*}(\mathbf{U})\right]=\mathbb{P}_{\mathbf{U}}\left[\mathbf{k}=\mathbf{k}^{*}(\mathbf{U})\right]$ which is the probability mass function of $\mathbf{K}^{*}$.

The motivation behind this relaxation is to take into account the local behaviour of the function around the conditional minimisers. For a given set of environmental 

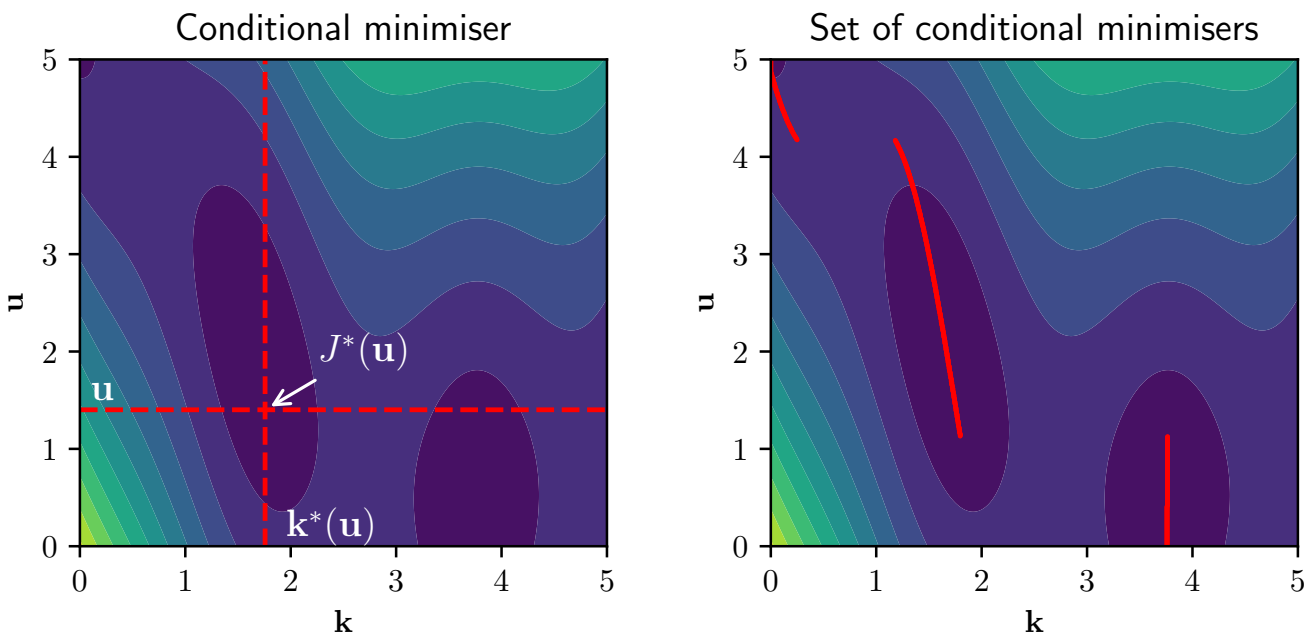

Relaxation of the constraint, $\alpha=1.5$
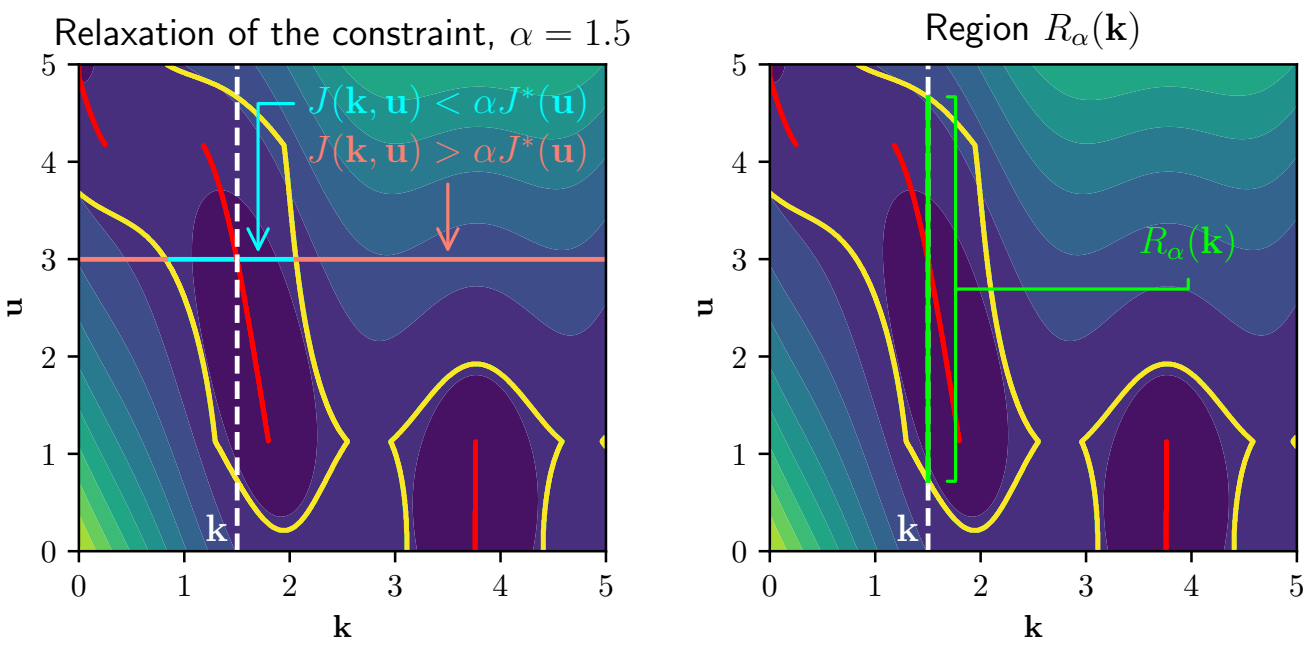

Figure 3: Principle of the relaxation of the constraint on $J_{\mathrm{BH}}$, and illustration of $R_{\alpha}(\mathbf{k})$. Top plots: Computation of the conditional minimisers $\mathbf{k}^{*}(\mathbf{u})$. Top right plot: the set $\left(\mathbf{k}^{*}(\mathbf{u}), \mathbf{u}\right)$ of conditional minimisers is represented in red. On the bottom left plot, for a relaxation $\alpha=1.5$, and $\mathbf{u}=3$, the acceptable $\mathbf{k}$ are in cyan, while the non-acceptable ones are in orange, while the frontier $\left\{\left(\mathbf{k}^{*}(\mathbf{u}), \mathbf{u}\right) \mid\right.$ $\left.J^{*}(\mathbf{u})=J(\mathbf{k}, \mathbf{U}=\mathbf{u})\right\}$ is in yellow. On the bottom right plot, the set $R_{\alpha}(\mathbf{k})$ for $\alpha=1.5$ and $\mathbf{k}=1.5$ is in green.

conditions $\mathbf{u}$, if the function $\mathbf{k} \mapsto J(\mathbf{k}, \mathbf{U}=\mathbf{u})$ is flat around its minimum $\mathbf{k}^{*}(\mathbf{u})$, then choosing $\mathbf{k}^{*}(\mathbf{u})+\epsilon$ (for a small $\epsilon$ ) will produce a value closer to the minimum than when the function has a high curvature. 
We consider in this work exclusively a multiplicative relaxation, instead of an additive one in the form $J(\mathbf{k}, \mathbf{u}) \leq J^{*}(\mathbf{u})+\beta$ for $\beta>0$. Both formulations of the regret: the additive $J-J^{*}$ and the relative $J / J^{*}$, take into account the value of the conditional minimum $J^{*}(\mathbf{u})$, but relative regret allow us to scale the region of acceptable points with respect to the best situation available: if $J^{*}(\mathbf{u})$ is close to 0 , the region of acceptable points will grow slowly, so at a given $\alpha$, we put emphasis on $\mathbf{k}^{*}(\mathbf{u})$. On the other hand, if $J^{*}(\mathbf{u})$ is large, it means that tuning the parameter to $\mathbf{k}^{*}(\mathbf{u})$ will not lead to very good performances of the cost function anyway. We can then put less weight on this value $\mathbf{k}^{*}(\mathbf{u})$ in the estimation, hence the region of acceptability that grows quickly. Finally, the relative regret is a normalized quantity, in constrast to the additive regret, which shares the same unit as $J$. This normalization, as well as the direct relation to the relative error $\frac{J-J^{*}}{J^{*}}$ allows for an interpretation of the relaxation as a fraction of error.

The choice of the relaxation constant $\alpha$ can be made to ensure the existence of a parameter that is "acceptable" with a certain probability. For instance, given that $J>0$, $\Gamma_{\alpha}(\mathbf{k})$ is increasing with respect to $\alpha$ for any $\mathbf{k} \in \mathbb{K}$. We can then focus on the smallest value of $\alpha$ such that $\Gamma_{\alpha}$ reaches a certain level of confidence $p \in[0,1]$. This leads to the definition of $\alpha_{p}$

$$
\begin{aligned}
\alpha_{p} & =\inf \left\{\alpha \geq 1 \mid \exists \mathbf{k}_{p} \in \mathbb{K}, \Gamma_{\alpha}\left(\mathbf{k}_{p}\right) \geq p\right\} \\
& =\inf \left\{\alpha \geq 1 \mid \max _{\mathbf{k} \in \mathbb{K}} \Gamma_{\alpha}(\mathbf{k}) \geq p\right\}
\end{aligned}
$$

Rewriting the equation above, we can express $\alpha_{p}$ as the solution of the following chance constrained problem

$$
\left\{\begin{array}{cl}
\min & q \\
\text { s.t. } & \max _{\mathbf{k}} \mathbb{P}_{\mathbf{U}}\left[\frac{J(\mathbf{k}, \mathbf{U})}{J^{*}(\mathbf{U})} \leq q\right] \geq p
\end{array}\right.
$$

that is the smallest $\alpha$, such that there exists a particular $\mathbf{k}_{p} \in \mathbb{K}$ for which $J\left(\mathbf{k}_{p}, \mathbf{U}\right) \leq$ $\alpha_{p} J^{*}(\mathbf{U})$ with probability $p$. As highlighted by the formulation of Eq. (20), $\mathbf{k}_{p}$ and $\alpha_{p}$ are the result of the optimisation of the Value-at-Risk of the random variable $J(\mathbf{k}, \mathbf{U}) / J^{*}(\mathbf{U})$, which is a measure of risk usually applied in the financial sector (see [37]). In this spirit, when choosing $\mathbf{k}_{p}$, the relative error of the function $J$ will be less than $\alpha_{p}$ with probability $p$. The maximal relative regret of the function will be $\alpha_{p}$, except for the $100(1-p) \%$ least favourable cases. 
The set of maximisers of $\Gamma_{\alpha_{p}}$ for different $p:\left\{\mathbf{k}_{p}\right.$ for $\left.p \in[0 ; 1]\right\}$ is what we are calling the relative-regret family of estimators RRE.

Figure 4 shows examples of $\Gamma_{\alpha_{p}}$ for $J_{\mathrm{BHswap}}$, at different levels $p$, and the associated estimates of the RRE. We can see that changing the level $p$ shifts the maximiser of $\Gamma_{\alpha_{p}}$. Since $\mathbf{k}_{1}$ is located quite far from the conditional minimisers, it arises as a compromise when the relaxation is large enough.

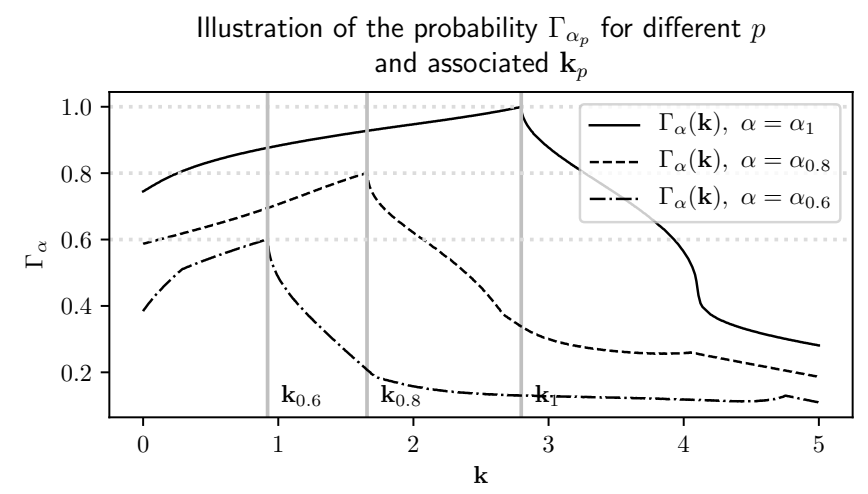

Figure 4: Illustration of the influence of different levels $p$ on $\Gamma_{\alpha_{p}}$ and on $\mathbf{k}_{p}$ for $J_{\mathrm{BHswap}}$. When $p$ increases, $\Gamma_{\alpha_{p}}$ increases as well.

We have then introduced the RRE, a family of estimators among which we can choose either large level of confidence $p$ i.e. a large robustness as we are controlling the relative regret for a large fraction of possible configurations, or we can look for almost optimal performances albeit for a more reduced number of situations. Those quantities require the evaluation and optimisation of probabilities, so solving such a problem can be quite challenging. Moreover, the choice of a member of the RRE, that is the choice of a level of confidence $p$ is also up to the modeller.

\subsection{Choice and computation of the relaxation coefficient}

\subsubsection{Almost-surely bounded relative-regret}

Let us first consider that we want to satisfy the chance constraint of Eq. (20) almost surely: this is the particular case of $p=1$. Given the strict positivity of $J$, the choice of such a level of confidence is possible only if there exists a $\mathbf{k}$ such that the ratio 
$J(\mathbf{k}, \mathbf{U}) / J^{*}(\mathbf{U})$ is bounded almost surely, and $\alpha_{1}$ is the solution of the (almost-surely) chance constrained problem:

$$
\left\{\begin{array}{cl}
\min & q \\
\text { s.t. } & \min _{\mathbf{k}} \frac{J(\mathbf{k}, \mathbf{U})}{J^{*}(\mathbf{U})} \leq q \quad \text { a.s. }
\end{array}\right.
$$

thus, $\mathbf{k}_{1}$ verifies

$$
\mathbb{P}_{\mathbf{U}}\left[\frac{J\left(\mathbf{k}_{1}, \mathbf{U}\right)}{J^{*}(\mathbf{U})} \leq \alpha_{1}\right]=1 \Longleftrightarrow \frac{J\left(\mathbf{k}_{1}, \mathbf{U}\right)}{J^{*}(\mathbf{U})} \leq \alpha_{1} \quad \text { a.s. }
$$

Under the assumption that $J(\mathbf{k}, \cdot)$ is continuous for all $\mathbf{k}$, this is equivalent to approaching this problem using uncertain sets to model the uncertain nature of $\mathbf{U}$ :

$$
\frac{J\left(\mathbf{k}_{1}, \mathbf{u}\right)}{J^{*}(\mathbf{u})} \leq \alpha_{1} \quad \forall \mathbf{u} \in \mathbb{U}
$$

it follows then that

$$
\alpha_{1}=\max _{\mathbf{u} \in \mathbb{U}} \frac{J\left(\mathbf{k}_{1}, \mathbf{U}=\mathbf{u}\right)}{J^{*}(\mathbf{u})}=\min _{\mathbf{k} \in \mathbb{K}}\left\{\max _{\mathbf{u} \in \mathbb{U}} \frac{J(\mathbf{k}, \mathbf{U}=\mathbf{u})}{J^{*}(\mathbf{u})}\right\}
$$

This can then be linked to Savage's minimax approach [38], which consists in a worstcase approach for the additive regret $J-J^{*}$, when in Eq. (24) we look to minimise the worst-case scenario in terms of the ratio.

Using Sample Average Approximation (SAA), based on $N$ i.i.d. samples of $\mathbf{U}:\left\{\mathbf{u}^{i}\right\}_{1 \leq i \leq N}$, we can reformulate the problem Eq. (21) as

$$
\left\{\begin{array}{cl}
\min & q \\
\text { s.t. } & \min _{\mathbf{k}} \frac{J\left(\mathbf{k}, \mathbf{u}^{\mathbf{i}}\right)}{J^{*}\left(\mathbf{u}^{\mathbf{i}}\right)} \leq q \quad \text { for } 1 \leq i \leq N
\end{array}\right.
$$

For a solution $\hat{\alpha}_{1}$ of Eq. (25), as SAA acts as a relaxation of the initial problem Eq. (21), the solution found acts as lower bound on the true value $\alpha_{1}: \hat{\alpha}_{1} \leq \alpha_{1}$.

Moreover, this estimated value $\hat{\alpha}_{1}$ can be used for the estimation of the relaxation constant for a level $p$ close to 1 . Using Clopper-Pearson intervals $[39,40]$, if $\hat{\alpha}_{1}$ is a solution of Eq. (25), it is also a feasible solution of Eq. (20) at a level $p=\left(\frac{\eta}{2}\right)^{1 / N}$ with probability $1-\eta$ : $\hat{\alpha}_{1}$ is then a probabilistic upper bound on $\alpha_{\left(\frac{\eta}{2}\right)^{\frac{1}{N}}}$.

$$
\hat{\alpha}_{1} \in\left[\alpha_{\left(\frac{\eta}{2}\right)^{1 / N}}, \alpha_{1}\right] \text { with probability } 1-\eta
$$


The estimated value $\hat{\alpha}_{1}$ will then be optimistic in the sense that it will underestimate the true value $\alpha_{1}$. We can however control this approximation using the probabilistic bound lower bound of Eq. (26)

Choosing a level of confidence $p=1$ suffers from the same pitfall as the worst-case approach, as it may return over-conservative solutions, provided that a solution exists in the first place.

\subsubsection{Estimation of $R R E$}

We are now going to focus on the more general case, where we can choose: to fix $p \leq 1$ and deduce $\alpha_{p}$, or to set a maximal threshold $\alpha$ and maximise the measure of the acceptable region, or finally to find a compromise $p$ and $\alpha_{p}$ in order to keep the latter not too large.

A first approach is to set $\alpha$, and then to estimate the function $\mathbf{k} \mapsto \Gamma_{\alpha}(\mathbf{k})$ as defined in Eq. (17). For instance, when $N$ samples from $\mathbf{U}$ are available, namely $\left\{\mathbf{u}^{i}\right\}_{1 \leq i \leq N}$, a possible estimate of $\Gamma_{\alpha}$ is

$$
\hat{\Gamma}_{\alpha}(\mathbf{k})=\frac{\operatorname{Card}\left\{\mathbf{u}^{i} \mid J\left(\mathbf{k}, \mathbf{U}=\mathbf{u}^{i}\right) \leq \alpha J^{*}\left(\mathbf{u}^{i}\right)\right\}}{N}=\frac{1}{N} \sum_{i=1}^{N} \mathbb{1}_{\left\{J\left(\mathbf{k}, \mathbf{U}=\mathbf{u}^{i}\right) \leq \alpha J^{*}\left(\mathbf{u}^{i}\right)\right\}}
$$

This expression is maximised with respect to $\mathbf{k}$, giving $\hat{p}=\max _{\mathbf{k}} \hat{\Gamma}_{\alpha}(\mathbf{k})$. We can also provide a confidence interval for the true value of $\Gamma_{\alpha}(\mathbf{k})$ where $\mathbf{k}=\arg \max \hat{\Gamma}_{\alpha}$.

However, unless the modeller has some precise idea of desired relaxation, doing so may lead to an unsatisfactory pairing of $\alpha$ and $p$. Indeed if $\alpha$ is chosen too small, the resulting $\hat{p}=\max _{\mathbf{k}} \hat{\Gamma}_{\alpha}(\mathbf{k})$ will be also small, meaning that the cost function will have non acceptable values with high probability.

Similarly, if $p$ is fixed, the corresponding $\hat{\alpha}_{p}$ is computed by searching for the smallest $\alpha$ satisfying $\max _{\mathbf{k}} \hat{\Gamma}_{\alpha}(\mathbf{k})=p$, or equivalently, by minimizing the quantile of order $p$ of the relative-regret, which is the ratio $J / J^{*}$. Once again, if $\hat{\alpha}_{p}$ is too large, the cost function may not be controlled enough for the contemplated application.

Looking for a compromise between $p$ and $\alpha$ would be preferable. This could be achieved by studying $p \mapsto \alpha_{p}$, and particularly its slope. If this curve presents a steep increase, the multiplicative constant $\alpha_{p}$ must be increased by a large amount in order to increase the probability $p$ by a small amount. Interesting couples $\left(p, \alpha_{p}\right)$ would then be the ones located before an abrupt increase of the slope of $p \mapsto \alpha_{p}$. 
Another possibility is to model this compromise by the ratio $\left(p / \alpha_{p}\right)$, as it increases with respect to $p$ and decreases with respect to $\alpha_{p}$. The level of confidence $p_{\text {ratio }}$ is then defined as the maximiser of $p \mapsto p / \alpha_{p}$.

\subsection{Numerical Illustration}

In this Section, we will compare the different estimators introduced previously and summarised in Table 1 on $J_{\mathrm{BH}}$ and $J_{\mathrm{BHswap}}$.

\begin{tabular}{lrl}
\hline Definition & Related quantities & Interpretation \\
\hline $\arg \min _{\mathbf{k} \in \mathbb{K}} \mathbb{E}_{\mathbf{U}}[J(\mathbf{k}, \mathbf{U})]$ & $\mathbf{k}_{\mathbb{E}}$ & Long run performances \\
$\arg \min _{\mathbf{k} \in \mathbb{K}} \operatorname{Var}_{\mathbf{U}}[J(\mathbf{k}, \mathbf{U})]$ & $\mathbf{k}_{\mathbb{V}}$ & Steady performances \\
$\arg \max _{\mathbf{k} \in \mathbb{K}} p_{\mathbf{K}^{*}}(\mathbf{k})$ & $\mathbf{k}_{\mathrm{MPE}}$ & Most probable minimiser \\
$\inf \left\{\alpha \mid \exists \mathbf{k}_{p} \in \mathbb{K}, \Gamma_{\alpha}\left(\mathbf{k}_{p}\right) \geq p\right\}$ & $\left(p, \mathbf{k}_{p}, \alpha_{p}\right)$ & Acceptable values \\
& & with fixed probability $p$ \\
$p_{\text {ratio }}=\arg \max p / \alpha_{p}$ & $\left(p_{\text {ratio }}, \mathbf{k}_{\text {ratio }}, \alpha_{\text {ratio }}\right)$ & Maximal ratio of $p$ and $\alpha_{p}$ \\
\hline
\end{tabular}

Table 1: Robust estimators, based on a cost function $J$

As stated before, we chose to model the uncertainties as a random variable uniformly distributed on $\mathbb{U}$. The bounded nature of $\mathbb{U}$ allows us to consider members of the RRE up to a level of confidence $p=1$. From now on, $\hat{\mathbf{k}}_{\mathrm{MPE}}$ is estimated using KDE with Gaussian kernels.

The smallest estimated relaxation $\hat{\alpha}_{1}$ and the corresponding $\hat{\mathbf{k}}_{1}$ has been computed for $J_{\mathrm{BH}}$ and $J_{\mathrm{BHswap}}$, using a regular grid of $1000 \times 1000$ points on $\mathbb{K} \times \mathbb{U}$. The contour plots of those functions can be seen in the top plots of Figure 5. The frontier corresponding to the couples of points $(\mathbf{k}, \mathbf{u})$ verifying $\left\{J(\mathbf{k}, \mathbf{U}=\mathbf{u})=\alpha J^{*}(\mathbf{u})\right\}$ has been drawn on top of these contour plots, for $\alpha=\hat{\alpha}_{1}$ and an arbitrary $\alpha=1.5<\hat{\alpha}_{1}$ to illustrate the effect of the acceptable region when the relaxation $\alpha$ changes. On the bottom plots, the curves $\mathbf{k} \mapsto \hat{\Gamma}_{\alpha}(\mathbf{k})$ for $\alpha=\hat{\alpha}_{1}$ and $\alpha=1.5$ along with the histograms of the minimisers are represented.

One can notice that the relaxation allows us to avoid the issue brought by the accumulation of the minimisers of $J_{\mathrm{BH}}$ at 3.8, as opposed to the MPE and its dependence on the estimation procedure of the distribution. 


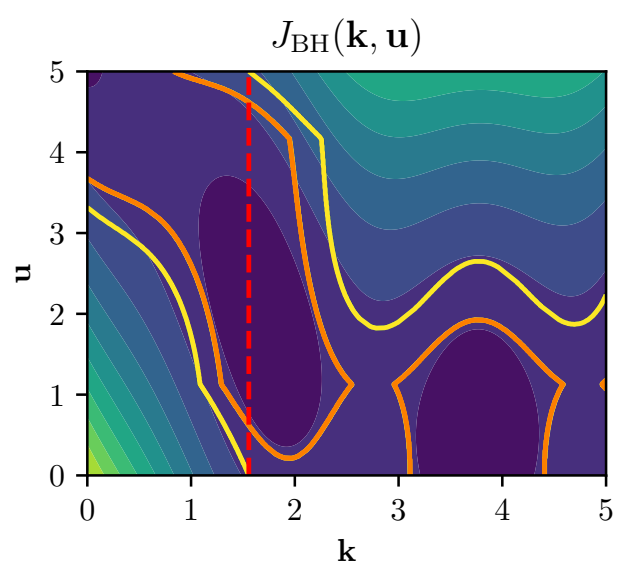

Probability $\hat{\Gamma}_{\hat{\alpha}_{1}}(\mathbf{k})$

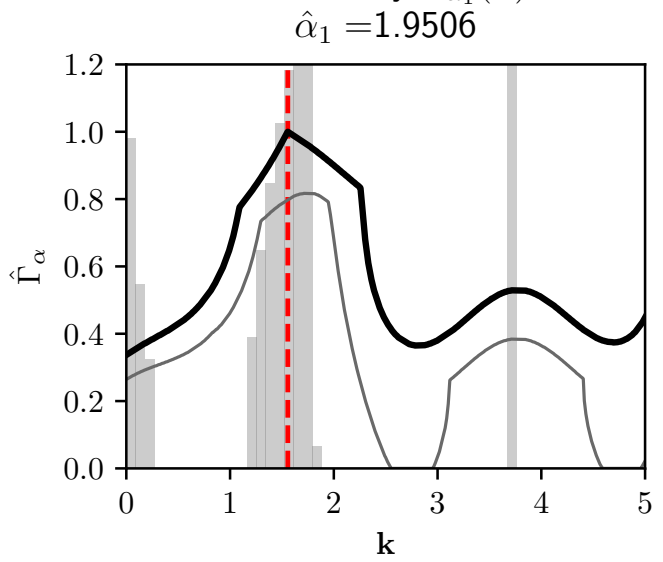

$-\hat{\Gamma}_{\hat{\alpha}_{1}}(\mathbf{k}) \quad---\hat{\mathbf{k}}_{1}$

$-\hat{\Gamma}_{1.5}(\mathbf{k}) \quad\left\{(\mathbf{k}, \mathbf{u}) \mid J(\mathbf{k}, \mathbf{u})=\hat{\alpha}_{1} J^{*}(\mathbf{u})\right\}$

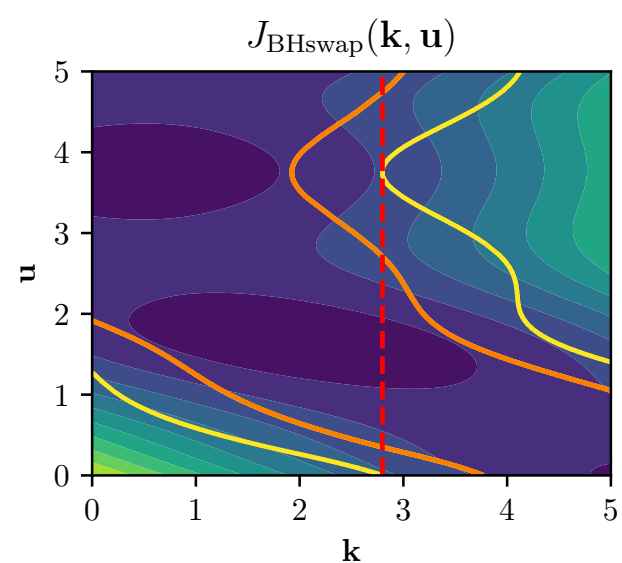

Probability $\hat{\Gamma}_{\hat{\alpha}_{1}}(\mathbf{k})$

$\hat{\alpha}_{1}=2.2196$

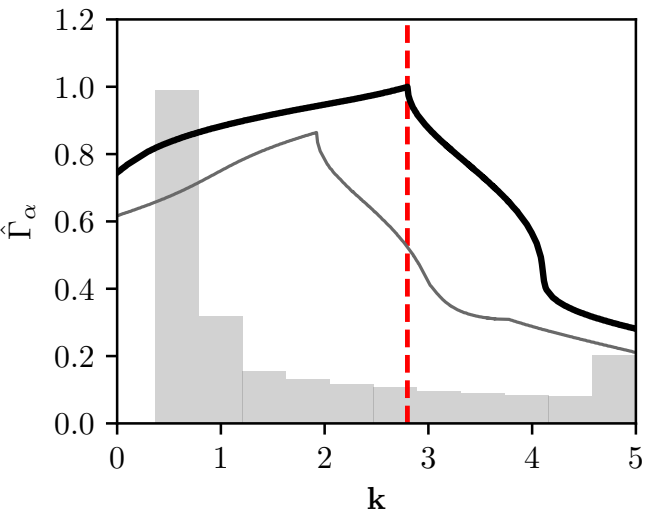

$\mathbf{k}$

Figure 5: Top: $J_{\mathrm{BH}}$ and $J_{\mathrm{BHswap}}$ contours. The thick yellow lines are the boundaries of the acceptable region defined for $\hat{\alpha}_{1}$, the thick orange are for $\alpha=1.5$. The red dashed line is the estimation $\hat{\mathbf{k}}_{1}$. Bottom: $\hat{\Gamma}_{\alpha}$ for $\alpha=1.5$ and $\alpha=\hat{\alpha}_{1}$, and estimated density of the minimisers.

In order to choose a satisfying level of confidence $p$, we are going to study $p \mapsto \hat{\alpha}_{p}$ and $p \mapsto p / \hat{\alpha}_{p}$, as described in Section 3.2.

The plot of $p \mapsto \hat{\alpha}_{p}$ for $J_{\mathrm{BH}}$ on Figure 6 shows what seems to be a piecewise linear behaviour. The last change of slope, i.e. for $p \approx 0.9$ corresponds to a local maximum of the ratio, while the first change of slope at $\hat{p}_{\text {ratio }}=0.654$ corresponds to the global 
maximum of the ratio. The RRE will then be evaluated for both of these values, as well as $p=1$ for reference.
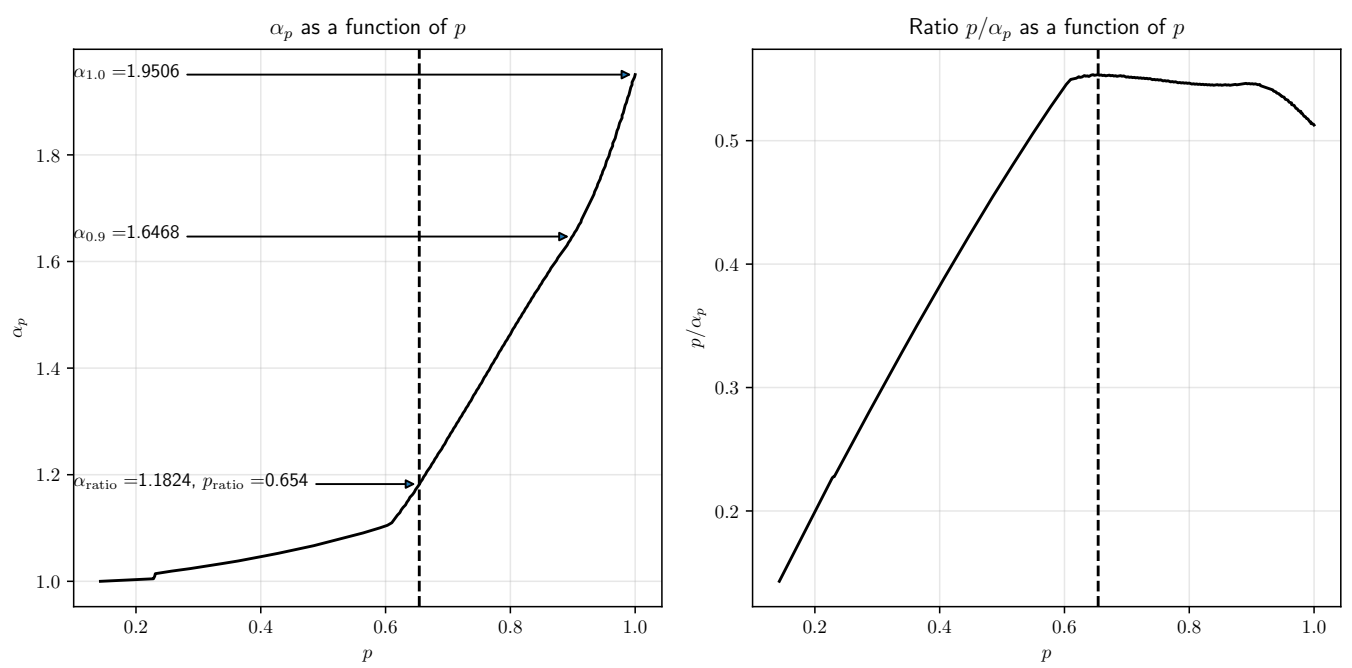

Figure 6: Evolution of the couples $\left(p, \alpha_{p}\right)$ and corresponding ratio $p / \alpha_{p}$ for $J_{\mathrm{BH}}$. The dashed line indicates the level $p$ associated with the highest ratio

For $J_{\mathrm{BH}}$, the numerical values of the robust estimators can be found in Table 2. For this particular problem, the different estimates are close to each other.

Table 2: Estimation performed for $J_{\mathrm{BH}}$, sorted by value

\begin{tabular}{ll} 
Estimate & Value \\
\hline$\hat{\mathbf{k}}_{\mathbb{V}}$ & 1.371 \\
$\hat{\mathbf{k}}_{p}, p=1$ & 1.557 \\
$\hat{\mathbf{k}}_{\mathbb{E}}$ & 1.587 \\
$\hat{\mathbf{k}}_{\mathrm{MPE}}$ & 1.628 \\
$\hat{\mathbf{k}}_{\text {ratio }}, \hat{p}_{\text {ratio }}=0.654$ & 1.637 \\
$\hat{\mathbf{k}}_{p}, p=0.90$ & 1.797 \\
\hline
\end{tabular}

Practically speaking, in order to compare the effective values taken by the objective function given an estimate $\hat{\mathbf{k}}$ we are going to consider the functions $\mathbf{u} \mapsto J(\hat{\mathbf{k}}, \mathbf{U}=\mathbf{u})$, that we will call "profiles of $\hat{\mathbf{k}}$ ". Those profiles are well suited for the representation of 
the cost function for an estimate $\hat{\mathbf{k}}$ fixed as the uncertain variable is modelled with a $1 \mathrm{D}$ uniform random variable.

For $J_{\mathrm{BH}}$, the curves are plotted in Figure 7 .

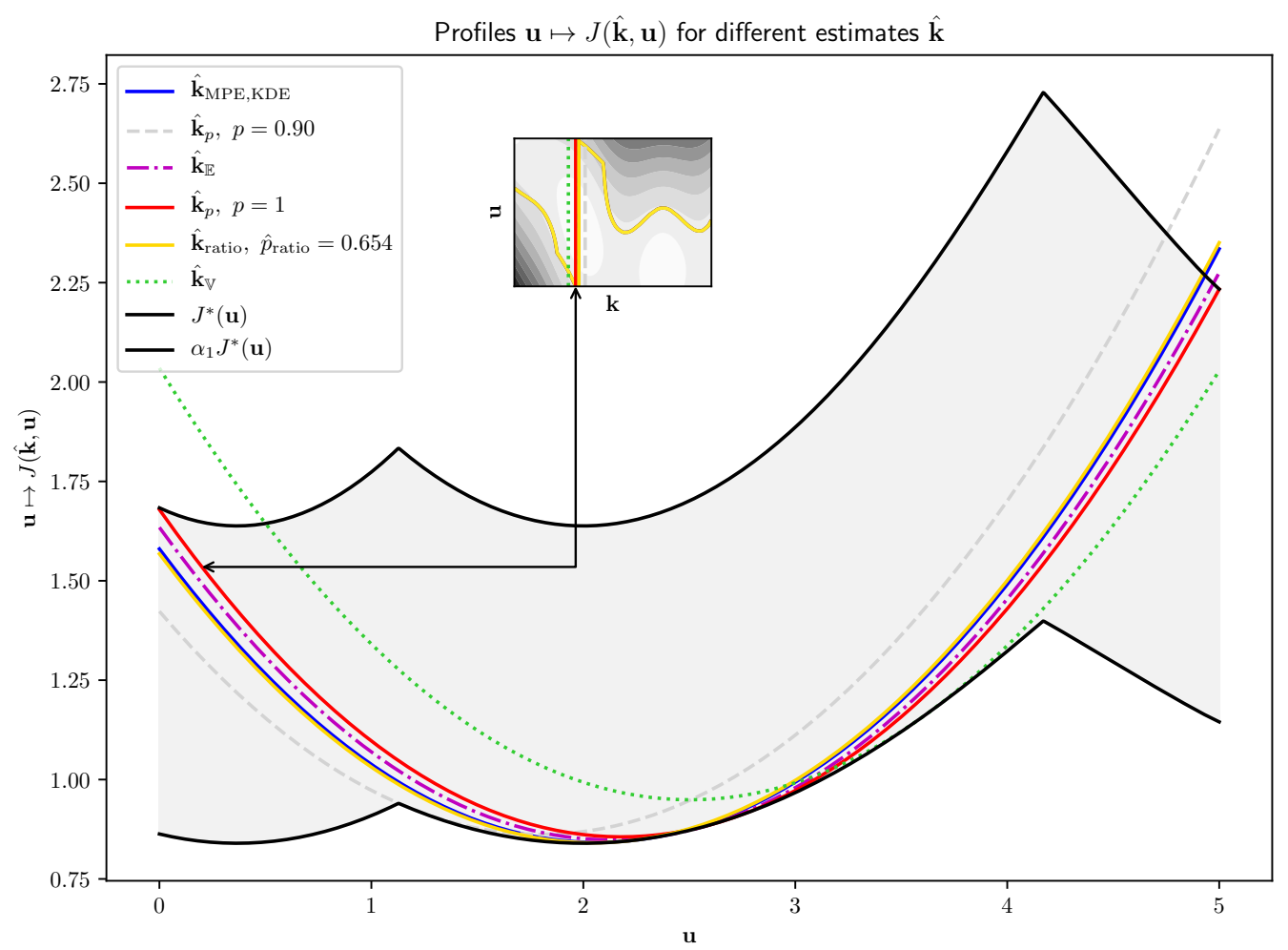

Figure 7: Profiles of the different estimates for $J_{\mathrm{BH}}$, corresponding to the vertical cross sections of the contour. The shaded region corresponds to the interval $\left[J^{*}(\mathbf{u}), \hat{\alpha}_{1} J^{*}(\mathbf{u})\right]$. The profiles of $\hat{\mathbf{k}}_{\mathrm{MPE}}$ and $\hat{\mathbf{k}}_{\text {ratio }}$ coincide in this case

By construction, the profile of $\hat{\mathbf{k}}_{1}$ is always within the shaded region, corresponding to $\left[J^{*}(\mathbf{u}), \hat{\alpha}_{1} J^{*}(\mathbf{u})\right]$. The profile of $\hat{\mathbf{k}}_{\mathbb{E}}$ in contrast, exceeds $\hat{\alpha}_{1} J^{*}(\mathbf{u})$ for $\mathbf{u}$ close to 5 , while the profile of $\hat{\mathbf{k}}_{\mathbb{V}}$ does it for $\mathbf{u}$ close to 0 . Except for $\hat{\mathbf{k}}_{\mathbb{V}}$, the different estimators give somewhat comparable results.

By contrast, $J_{\mathrm{BHswap}}$ will show a different behaviour as Figure 8 provides the plots of $p \mapsto \hat{\alpha}_{p}$ and $p \mapsto p / \hat{\alpha}_{p}$.

Compared to the similar plots for $J_{\mathrm{BH}}$ in Figure $6, \hat{\alpha}_{p}$ exhibits a smoother behaviour 

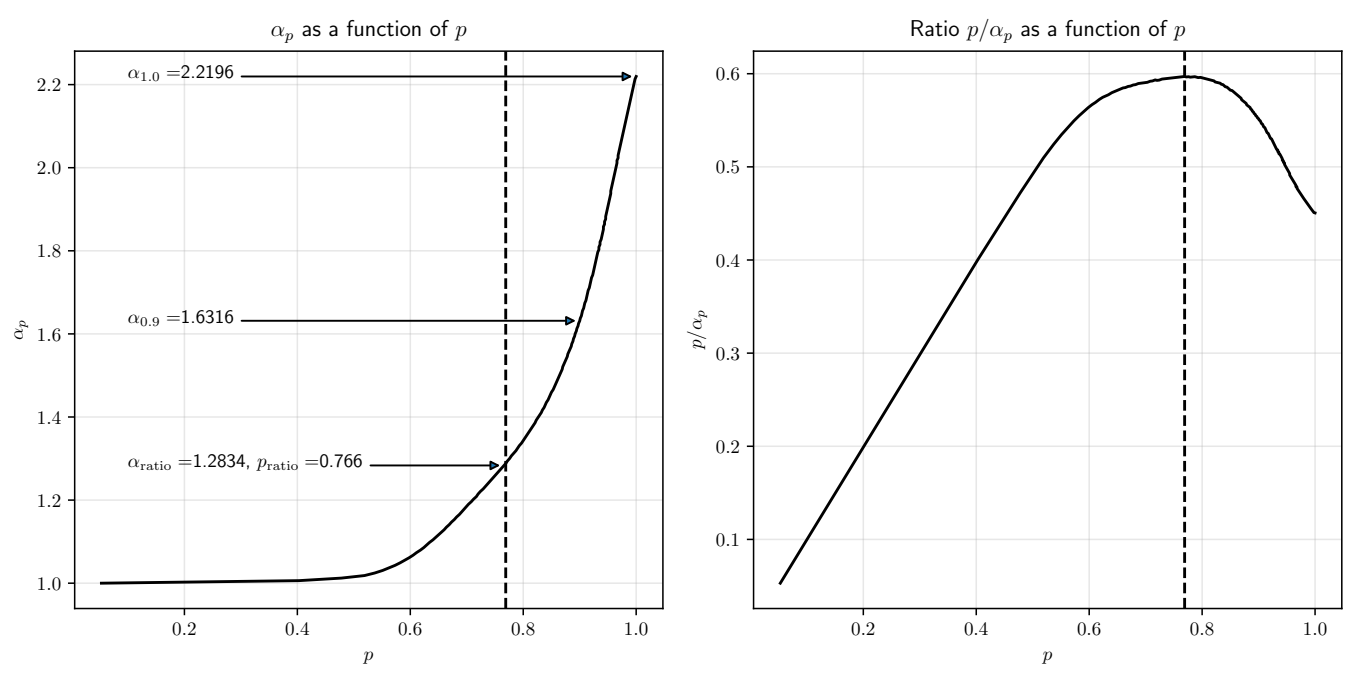

Figure 8: Evolution of the couples $\left(p, \alpha_{p}\right)$ and corresponding ratio $p / \alpha_{p}$ for $J_{\mathrm{BHswap}}$. The dashed line indicates the level $p$ associated with the highest ratio

for $J_{\mathrm{BHswap}}$ as no abrupt change of slope is easily discernable and the ratio presents a unique maximum for $\hat{p}_{\text {ratio }}=0.766$. The numerical values of the estimations $\hat{\mathbf{k}}$ presented in Table 3 show that, contrary to $J_{\mathrm{BH}}$, the calibrated values are more spread over $\mathbb{K}$.

\begin{tabular}{|c|c|}
\hline Estimate & Value \\
\hline$\hat{\mathbf{k}}_{\mathrm{MPE}}$ & 0.606 \\
\hline$\hat{\mathbf{k}}_{\text {ratio }}, \hat{p}_{\text {ratio }}=0.766$ & 1.537 \\
\hline$\hat{\mathbf{k}}_{\mathbb{E}}$ & 1.752 \\
\hline$\hat{\mathbf{k}}_{\mathbb{V}}$ & 2.638 \\
\hline$\hat{\mathbf{k}}_{1}, p=1$ & 2.798 \\
\hline
\end{tabular}

Profiles of the different estimates of $J_{\text {BHswap }}$ are shown in Figure 9.

In this case, $\hat{\mathbf{k}}_{\mathrm{MPE}}, \hat{\mathbf{k}}_{\mathbb{E}}$ and $\hat{\mathbf{k}}_{\text {ratio }}$ present a similar behaviour. They perform very well for $\mathbf{u}>2$, especially for $\hat{\mathbf{k}}_{\mathrm{MPE}}$ which is very close to the minimal value; however for $\mathbf{u}<2$, they produce high values of the function. The performances of $\hat{\mathbf{k}}_{1}$ are closer to the performances of $\hat{\mathbf{k}}_{\mathbb{V}}$ for this function, but it performs worse than $\hat{\mathbf{k}}_{\mathbb{E}}$ and $\hat{\mathbf{k}}_{\mathbb{V}}$ for 


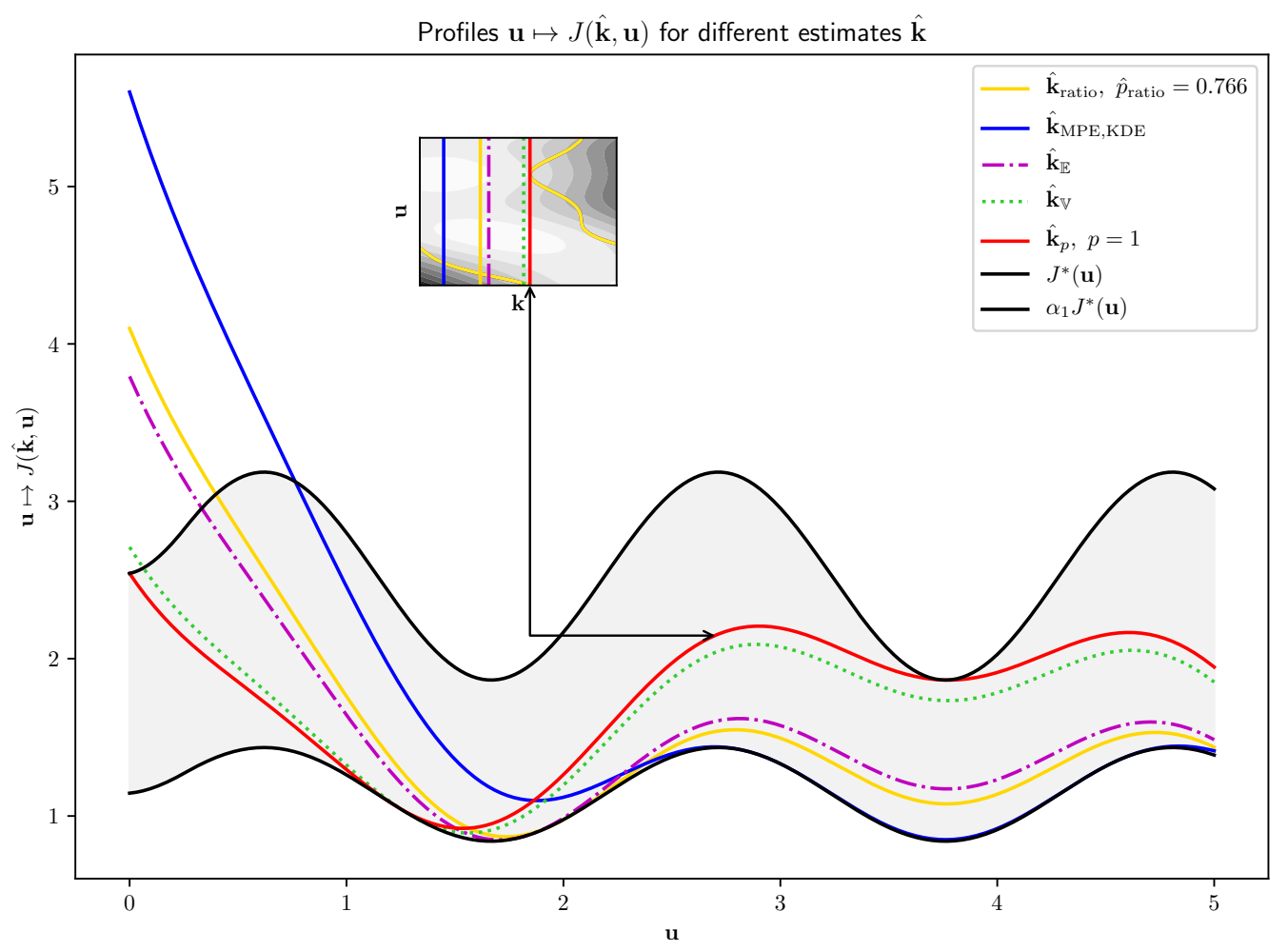

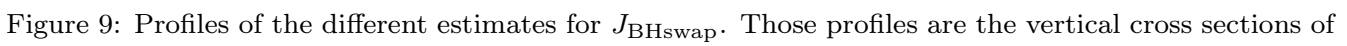
the contours above. The shaded region corresponds to the interval $\left[J^{*}(\mathbf{u}), \hat{\alpha}_{1} J^{*}(\mathbf{u})\right]$

$\mathbf{u}>2$, even though its range is designed to stay within the interval $\left[J^{*}(\mathbf{u}) ; \hat{\alpha}_{1} J^{*}(\mathbf{u})\right]$.

We have seen how some classical robust estimators and the RRE behave on two different analytical problems. In addition to the usual levels of confidence such as $90 \%$ and $95 \%$, one can also settle for an ad-hoc compromise, where $p$ maximises the ratio $p / \alpha_{p}$. When the sample space of $\mathbf{U}$ is bounded, a conservative solution is to set $p=1$. We are now going to see how can robust minimisation is applied on the calibration of a numerical model. 


\section{Robust calibration of a numerical model}

\subsection{Calibration of a toy numerical model}

We will follow the approach described in [41] in order to establish the function $\mathcal{G}$ described in the first section in Eq. (1), and the resulting cost function $J$.

The calibration of a numerical model is usually based on the comparison between the numerical model and some observations, during a fixed time interval $[0, T]$ called assimilation window. The modelled physical system can be seen as a map from $\mathbb{U}$ to $\mathbb{Y}$, the space of observations, denoted as $\mathcal{M}^{o}: \mathbf{u} \mapsto \mathcal{M}^{o}(\mathbf{u})$, where $\mathbf{u} \in \mathbb{U}$ is an input representing some environmental conditions. The observation mentioned above is the output of the physical system during the time-window, and is denoted by $\mathcal{M}^{o}\left(\mathbf{u}^{\text {true }}\right) \in \mathbb{Y}$, where $\mathbf{u}^{\text {true }} \in \mathbb{U}$ is unknown.

In addition of $\mathbf{u}$, the numerical model $\mathcal{M}$ depends on some other input $\mathbf{k} \in \mathbb{K}$. This additional parametrization comes usually from the successive simplifications needed to implement a numerical model of the observed physical system. $\mathbf{k}$ needs to be calibrated accordingly, so that the numerical model can be used to predict the behaviour of the physical system under different operating conditions.

The misfit $\mathcal{G}$ is defined as the difference between the numerical model and the observation. Choosing a squared norm, the cost function $J$ defined in Eq. (1) is

$$
J(\mathbf{k}, \mathbf{U}=\mathbf{u})=\frac{1}{2}\|\mathcal{G}(\mathbf{k}, \mathbf{u})\|^{2}=\frac{1}{2}\left\|\mathcal{M}(\mathbf{k}, \mathbf{u})-\mathcal{M}^{o}\left(\mathbf{u}^{\text {true }}\right)\right\|^{2}
$$

\subsection{The Shallow Water equations}

The model to calibrate is an implementation of the 1D Shallow Water equations, described in Eq. (29), where $h$ is the height of the water column, $q$ the discharge, and $z$ the bathymetry, while $g$ is the usual gravitation constant. The parameter to calibrate $\mathbf{k}$ is the quadratic friction term, proportional to the square of the inverse of ManningStrickler coefficient. The environmental parameter $\mathbf{u}$ is the amplitude of a sine wave of period $1 / \omega_{0}$. The domain of those two parameters are $\mathbb{K}=[0.0,1.3]$ and $\mathbb{U}=[0.5,0.7]$. 


$$
\left\{\begin{aligned}
\partial_{t} h+\partial_{x} q & =0 \\
\partial_{t} q+\partial_{x}\left(\frac{q^{2}}{h}+\frac{g}{2} h^{2}\right) & =-g h \partial_{x} z-\mathbf{k} q|q| h^{-7 / 3} \\
h(0, t) & =20.0+3 \cdot \sin \left(\frac{2 \pi t}{2}\right)+1.5 \cdot \mathbf{u} \cdot \sin \left(\frac{2 \pi t}{\omega_{0}}\right) \\
\partial_{x} q(0, t) & =0
\end{aligned}\right.
$$

These equations are integrated using a finite-volume scheme on a discretized domain $[0, L]$, up to a time $T$. The output of the computer code is the sea surface height $h$, on the center of all the volumes and at all the time-steps, that will be denoted $\mathcal{M}\left(\mathbf{k}, \mathbf{u} ; \omega_{0}=1.0\right)$. In this setting, the random variable $\mathbf{U}$ is uniformly distributed on $\mathbb{U}$.

To generate the observation, we set $\mathbf{u}^{\text {true }}=2 / 3$, and define $\mathcal{M}^{o}$ based on the computer model $\mathcal{M}$, such that $\mathcal{M}^{o}\left(\mathbf{u}^{\text {true }}\right)=\mathcal{M}\left(\mathbf{k}^{\text {true }}, \mathbf{u}^{\text {true }} ; \omega_{0}=0.999\right)$. $\omega_{0}$ represents here the uncontrollable error between the observations and the numerical model and will now be omitted systematically in the notation.

The true value of the bottom friction $\mathbf{k}^{\text {true }}=\left(k_{1}^{\text {true }}, k_{2}^{\text {true }}, \ldots, k_{N_{\text {vol }}}^{\text {true }}\right)$ is not constant over the whole domain, and is defined as

$$
k_{i}^{\text {true }}=0.2 \cdot\left(1+\sin \left(\frac{2 \pi x_{i}}{L}\right)\right)
$$

where $x_{i}$ is the center of the $i$-th volume. The two sources of systematic errors are the one-dimensionality of $\mathbb{K}$, and $\omega_{0}$. Given this setting, there exists no couple $(\mathbf{k}, \mathbf{u}) \in \mathbb{K} \times \mathbb{U}$ reproducing exactly the observations, thus the cost function will always be strictly positive.

\subsection{Computation of the robust estimates}

As the numerical model is expensive to run, it has first been evaluated on a relatively small regular grid covering $\mathbb{K} \times \mathbb{U}$, and a metamodel based on Gaussian processes is constructed using those initial evaluations. In order to better capture the locus of the conditional minimisers $\left\{\left(\mathbf{k}^{*}(\mathbf{u}), \mathbf{u}\right) \mid \mathbf{u} \in \mathbb{U}\right\}$, we select points maximising the PEI criterion $[42,43]$ in order to add points to the design that improve the metamodel accuracy close to the conditional minimisers. Afterwards, a bigger regular grid is evaluated by the metamodel once the design space has been sufficiently explored, and these computations are used to calibrate the model. 
The different steps of the estimation are illustrated Figure 10, where the top plot shows the contour plot of $J$, with the conditional minimisers and the RRE of level $p=1$. On the middle plot are shown the conditional moments, $\hat{\mathbf{k}}_{\mathbb{E}}$ and $\hat{\mathbf{k}}_{\mathbb{V}}$ and the estimated density of the minimisers. At the bottom of the Figure, $\hat{\Gamma}_{\alpha_{p}}$ are represented for different levels $p$. As $\mathbb{U}$ is bounded, $p=1$ is attainable, and $\hat{\mathbf{k}}_{1}$ is evaluated.
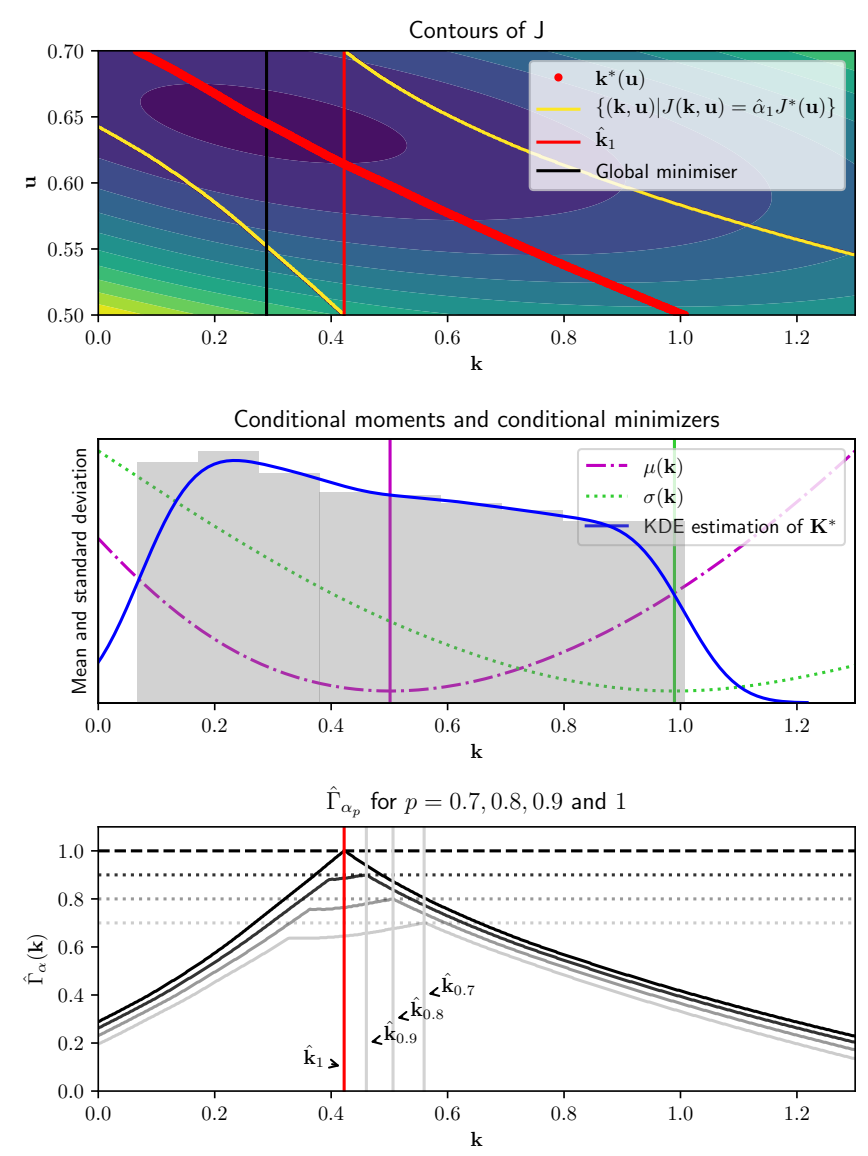

Figure 10: Procedure of robust calibration for the shallow water problem. Top: contours of $J$, conditional minimisers and $\left\{(\mathbf{k}, \mathbf{U}=\mathbf{u}) \mid J(\mathbf{k}, \mathbf{u})=\hat{\alpha}_{1} J^{*}(\mathbf{u})\right\}$. Middle: Conditional moments and histogram and KDE of the conditional minimisers. Bottom: $\hat{\Gamma}_{\alpha_{p}}$ for different levels $p$

We can see that the different estimations seem less problematic for this problem than for the analytical examples shown in Section 3.3, as everything appears to be very smooth and unimodal. 
Looking at $p \mapsto \alpha_{p}$ and $p \mapsto p / \alpha_{p}$ on Figure 11, we can see that $\alpha_{p}$ evolves almost linearly for $p>0.5$, and that the ratio $p / \alpha_{p}$ is monotonically increasing, so that the maximal ratio is found for $p=1$.
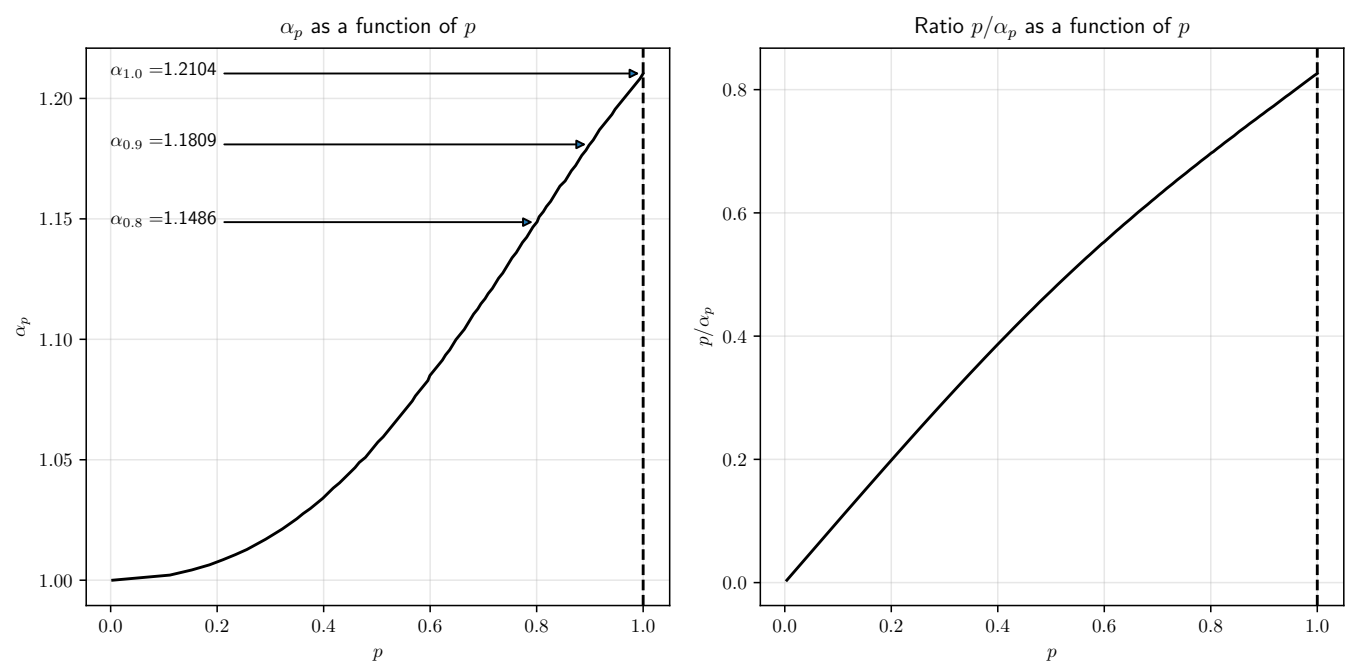

Figure 11: Evolution of $\alpha_{p}$ for different levels $p$, and ratio, for the shallow water problem

The different estimates take a wide range of values, as seen on Table 4, from 0.249 to almost 1.0 , while $k_{i}^{\text {true }} \in[0,0.4]$ for $1 \leq i \leq N_{\text {vol }}$. It can be noted that the calibration may lead to values outside the range given by the true ones. This can be interpreted as the fact that the calibration look to compensate for errors on $\omega_{0}$. As a basis for comparison, the global minimiser of $J$ over $\mathbb{K} \times \mathbb{U}$ has also been computed, and $\hat{\mathbf{k}}_{\text {global }}$ is then obtained by discarding the $\mathbf{u}$ value.

Similarly as for $J_{\mathrm{BH}}$ and $J_{\mathrm{BHswap}}$, the profiles are depicted Figure 12.

We can see that the performances of $\hat{\mathbf{k}}_{1}$ and $\hat{\mathbf{k}}_{\mathbb{E}}$ are very similar, but $\hat{\mathbf{k}}_{1}$ has better performances when $\mathbf{u}>0.6$. When comparing with the global optimiser $\hat{\mathbf{k}}_{\text {global }}, \hat{\mathbf{k}}_{1}$ performs better when $\mathbf{u}<0.625$, so more than half of the time. We are now going to compare how well some of those calibrated values compare in a forecast context.

\subsection{Assessing the quality of the forecast of the calibrated model}

For the calibration, the model has been integrated on a time-period $[0, T]$, called assimilation window, and have been compared with the observation of the sea water 
Table 4: Calibrated values of $\mathbf{k}$ according to different criteria, for the shallow water problem

\begin{tabular}{ll} 
Estimate & Value \\
\hline$\hat{\mathbf{k}}_{\mathrm{MPE}}$ & 0.249 \\
$\hat{\mathbf{k}}_{\text {global }}$ & 0.290 \\
$\hat{\mathbf{k}}_{p}, p=1$ & 0.423 \\
$\hat{\mathbf{k}}_{p}, p=0.90$ & 0.458 \\
$\hat{\mathbf{k}}_{\mathbb{E}}$ & 0.501 \\
$\hat{\mathbf{k}}_{p}, p=0.80$ & 0.505 \\
$\hat{\mathbf{k}}_{p}, p=0.70$ & 0.560 \\
$\hat{\mathbf{k}}_{\mathbb{V}}$ & 0.990 \\
\hline
\end{tabular}

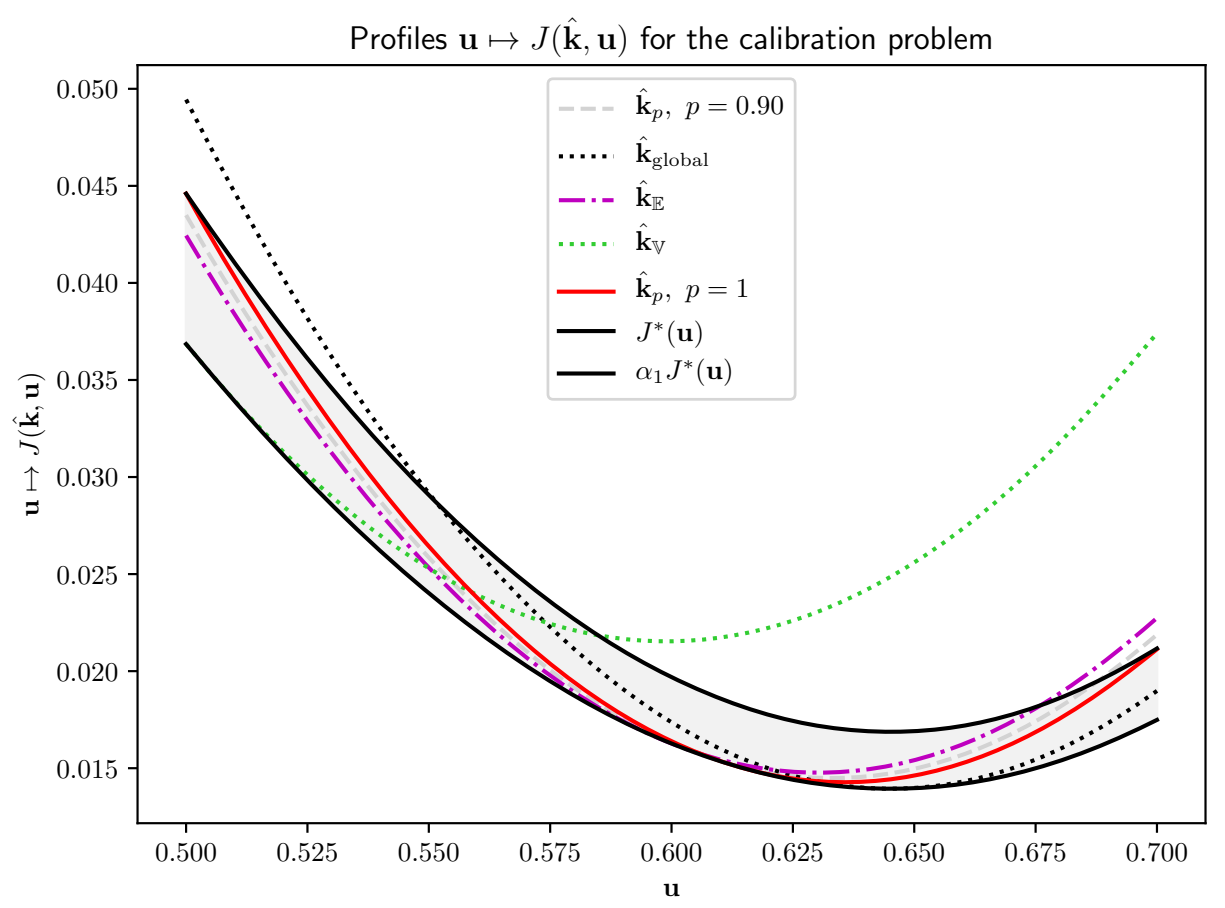

Figure 12: Profiles for the calibration problem, for the shallow water case

height on the same time-period. We now want to compare the quality of the different forecasts originating from different calibrated bottom frictions. Those forecasts result 
from the integration of the numerical model between the time $T$ and a time $T_{\text {pred }}$ : the forecast window.

Given the probabilistic nature of the environmental conditions $\mathbf{U}$, the forecasts will also be probabilistic. We will then compare $\mathcal{M}_{\text {pred }}^{o}(\mathbf{U})$ and $\mathcal{M}_{\text {pred }}(\mathbf{k}, \mathbf{U})$, that are the observation, and the numerical model on the forecast window for a calibrated $\mathbf{k}$.

Two metrics will be computed: the squared forecast error, and the Continuous Ranked Probability Score (CRPS) [44]. The parameters satisfying a robust criterion that are compared are the minimiser of the expectation $\hat{\mathbf{k}}_{\mathbb{E}}$, the minimiser of the variance $\hat{\mathbf{k}}_{\mathbb{V}}$ and the relative regret of level $p=1, \hat{\mathbf{k}}_{1}$ as described in the previous section. We will also feature the global minimiser $\hat{\mathbf{k}}_{\text {global }}$, and the conditional minimisers $\hat{\mathbf{k}}^{*}\left(\mathbf{u}^{i}\right)$ where the chosen environmental variables are $\left\{\mathbf{u}^{i}\right\}_{1 \leq i \leq 4}=\{0.5,0.55,0.65,0.7\}$. Those values, even though they do not meet a robustness criterion, are introduced in order to have a more precise idea on the possible performances of a deterministic version of the calibration problem. The conditional minimiser $\hat{\mathbf{k}}^{*}(0.6)$ has been omitted as it results in a value very similar to the minimum of the expectation.

\subsubsection{Squared forecast error}

A first simple approach to measure the forecast quality is to take the squared difference between the numerical model and the observation for two samples of $\mathbf{U}$. Given two environmental conditions $\mathbf{u}$ and $\mathbf{u}^{\prime} \in \mathbb{U}$, the former used to run the computer simulation and the latter to generate the observations, the squared forecast error for the parameter $\mathbf{k}$ is

$$
S_{\text {pred }}\left(\mathbf{k}, \mathbf{u}, \mathbf{u}^{\prime}\right)=\left(\mathcal{M}_{\text {pred }}(\mathbf{k}, \mathbf{u})-\mathcal{M}_{\text {pred }}^{o}\left(\mathbf{u}^{\prime}\right)\right)^{2}
$$

Averaging over both $\mathbf{u}$ and $\mathbf{u}^{\prime}$ defines the mean squared forecast error, defined on every point of the spatial domain, and at every time-steps. This can be done using a Monte-Carlo approximation. As $\mathbf{u}$ and $\mathbf{u}^{\prime}$ are i.i.d., assuming that we have a set of samples $\left\{\mathbf{u}^{i}\right\}_{1 \leq i \leq N_{\mathbf{u}}}$, the squared forecast error can then be approximated:

$$
S(\mathbf{k})=\frac{1}{N_{\mathbf{u}}^{2}} \sum_{i=1}^{N_{\mathbf{u}}} \sum_{j=1}^{N_{\mathbf{u}}} S_{\text {pred }}\left(\mathbf{k}, \mathbf{u}^{i}, \mathbf{u}^{j}\right)=\frac{1}{N_{\mathbf{u}}^{2}} \sum_{i=1}^{N_{\mathbf{u}}} \sum_{j=1}^{N_{\mathbf{u}}}\left(\mathcal{M}_{\text {pred }}\left(\mathbf{k}, \mathbf{u}^{i}\right)-\mathcal{M}_{\text {pred }}^{o}\left(\mathbf{u}^{j}\right)\right)^{2}
$$


The mean squared forecast error averaged over the whole space and over all the timesteps gives an indication on the overall prediction quality of the prediction given this metric, and are represented on the right of Figure 13. We can see that $\hat{\mathbf{k}}_{\text {global }}$ performs slightly better than $\hat{\mathbf{k}}_{1}$, itself performing slightly better than $\hat{\mathbf{k}}_{\mathbb{E}}$. Averaging $S(\hat{\mathbf{k}})$ over the time steps between $T$ and $T_{\text {pred }}$, we have an indication on the quality of the forecast in the squared sense depending on the spatial coordinate $x$, as seen on the left of Figure 13.
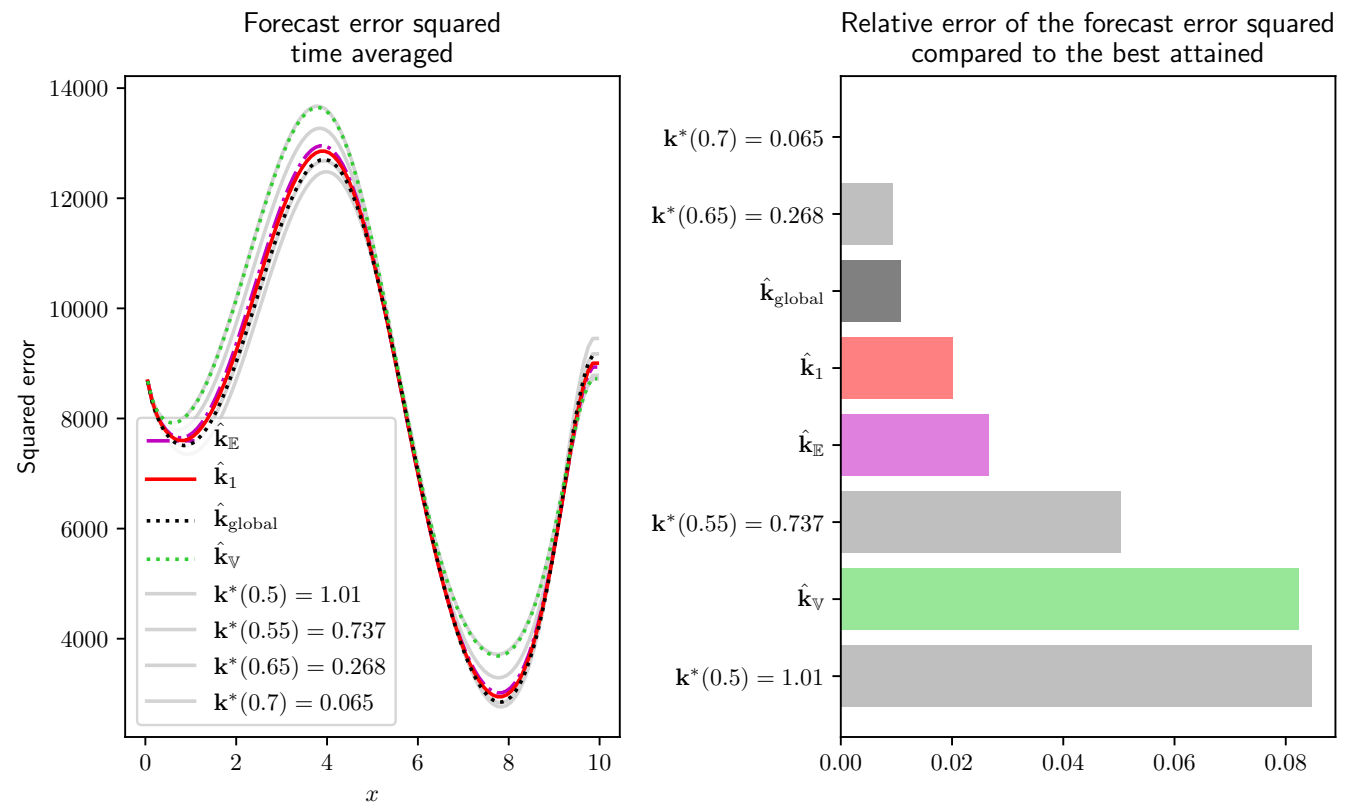

Figure 13: Squared forecast error for the shallow water case, depending on the calibrated parameter. The left figure shows the squared error time-averaged as a function of the spatial coordinate $x$, while the right barplot shows the relative change of the mean forecast error, averaged over time and space, taken w.r.t. to the best attained performance for $\mathbf{k}^{*}(0.7)$

We can see that the mean forecast error squared, on the right side, averaged over time and space is the smallest for the conditional minimisers $\hat{\mathbf{k}}^{*}(0.7)$ and $\hat{\mathbf{k}}^{*}(0.65)$, then $\hat{\mathbf{k}}_{\text {global }}$, while $\hat{\mathbf{k}}_{1.0}$ performs slightly better than $\hat{\mathbf{k}}_{\mathbb{E}}$.

It may seem surprising that some parameters calibrated without a robustness aspect perform better than $\hat{\mathbf{k}}_{1}$ and $\hat{\mathbf{k}}_{\mathbb{E}}$, but their performances are largely dependent on the choice of $\mathbf{u}$ and their associated conditional minimisers. Good forecasts can be achieved 
as well as bad ones, as shown for $\mathbf{u}=0.5$, that leads to bad forecasts.

One issue with the squared forecast error is that it will penalize strongly the forecasts that present high variability with respect to the environmental conditions. A way to deal with this is to use another metric, that takes the probabilistic nature of the forecasts into account.

\subsubsection{Continuous Ranked Probability Score}

Given the random variables $\mathcal{M}_{\text {pred }}(\hat{\mathbf{k}}, \mathbf{U})$ and $\mathcal{M}_{\text {pred }}^{o}(\mathbf{U})$, representing the probabilistic forecast and the probabilistic observations, we can define the cumulative distribution functions $(\mathrm{CDF}) F_{\text {pred }}(\cdot, \hat{\mathbf{k}})$ and $F_{\text {pred }}^{o}(\cdot)$ The Continuous ranked probability score (CRPS) measures the squared difference between the predicted CDF $F_{\text {pred }}$ using a calibrated value, and the CDF of the observations $F_{\text {pred }}^{o}$.

$$
\operatorname{CRPS}(\mathbf{k})=\int_{\mathbb{R}}\left(F_{\text {pred }}(\xi, \mathbf{k})-F_{\text {pred }}^{o}(\xi)\right)^{2} \mathrm{~d} \xi
$$

The left plot of Figure 14 shows the CRPS averaged over time, where $x$ denotes the spatial coordinate and the right plot shows the value of the CRPS averaged over time and space. The difference between the squared forecast error and the CRPS is apparent when comparing the general trends shared by the different calibrated parameters. According to the squared error, the sea water height of the physical region $x=4$ is not well predicted, while around $x=8$, the predictions are better. On the other hand, according to the CRPS, the region around $x=8$ provide worse forecasts than when $x=4$ and $x=7$. Given the properties of the two metrics, we can conclude that the region around $x=4$ presents a lot of variability with respect to $\mathbf{u}$, for both the true model and the numerical one. However, for $x \approx 8$, there is a lot less variability, as the low squared error indicates, but probably a higher bias, due to the systematic errors between the truth and the numerical model.

The numerical evaluations of the CRPS for different parameters show the same order of performances observed for the squared error: the calibrated parameters that present the best performances for forecasts according to those two metrics are $\hat{\mathbf{k}}^{*}(0.7), \hat{\mathbf{k}}^{*}(0.65)$ which is very similar to $\hat{\mathbf{k}}_{\text {global }}$, and then $\hat{\mathbf{k}}_{1}$, and $\hat{\mathbf{k}}_{\mathbb{E}}$. This presupposes to know which $\mathbf{u}$ to choose for the conditional minimisation, thus having a strong insight on the value of the parameters in the first place. 

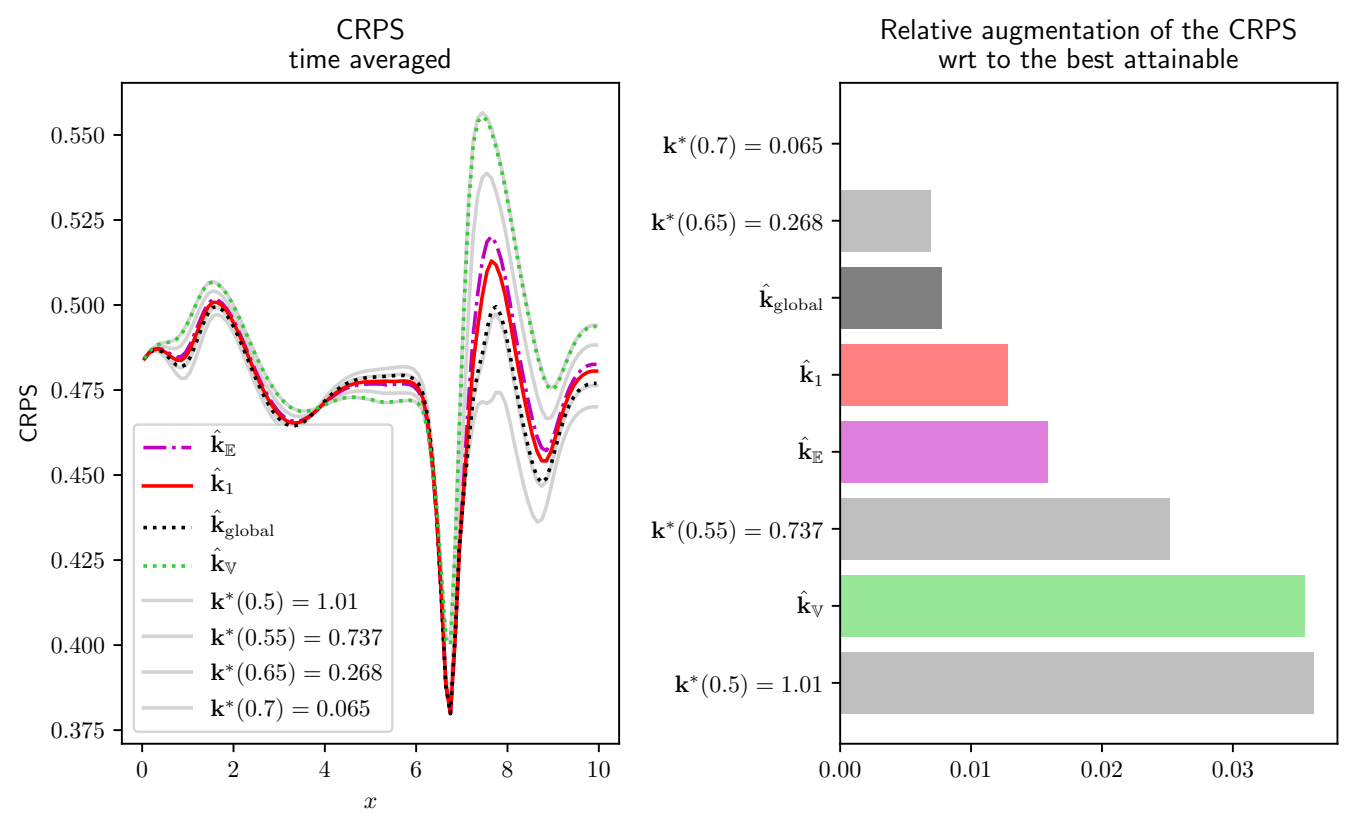

Figure 14: CRPS computed for different calibrated parameters for the shallow water case. The left figure shows the CRPS time-averaged as a function of the spatial coordinate $x$, and the right figure shows the relative change of the CRPS averaged over time and space, taken with respect to the best attained CRPS for $\mathbf{k}^{*}(0.7)$

\section{Conclusion}

In this paper, we dealt with a problem of robust calibration, or in other terms, the robust minimisation of an objective function. To adress this issue, we proposed a new set of robust estimators: the family of the RRE (Relative-regret estimators), and compared it in a forecast context to some other robust estimators, for the calibration of the bottom friction of a shallow water model.

Our approach is based on the comparison of the objective function to the optimal value it can attain, given a realisation of the uncertain variable. By constraining this ratio in terms of probability, the modeller can then minimize the frequency at which the relative error of the objective function will exceed a prescribed threshold. Alternatively, at a level of confidence $p$, the associated threshold $\alpha_{p}$ bounds the relative deviation of the objective function for the proportion $p$ of the most favourable cases. The resulting 
estimate can then be assessed robust, as it corresponds to values of the objective function as close as possible to the optimal values.

Except for simple analytical examples, the exact evaluation of a member of the RRE is very expensive computer-wise, not to say impossible. Some bottlenecks appear: the computations of the conditional minimisers $\mathbf{k}^{*}(\mathbf{u})$ and the minimum $J^{*}(\mathbf{u})$, that require very local exploration of $\mathbb{K}$ for every $\mathbf{u}$; and the computations of the probability of overshooting a given bound that depends on $J^{*}(\mathbf{u})$ (analogous to a probability of failure), task that requires an efficient exploration of the whole sample space $\mathbb{U}$.

For the calibration of the shallow water model presented in Section 4, we chose to construct a response surface based on Gaussian processes, which is then used for the extensive computations. This construction is first based on an initial design, that is enriched to better capture the locus of the conditional minimisers using the PEI criterion introduced in [42].

Practically speaking, the estimation of those quantities is very crude, so the computational cost may be very expensive. As perspective, specific strategies have to be created in order to carefully select each additional point that will be evaluated, and thus reduce the amount of evaluations needed of the numerical model. This selection can be based for instance on sequential design of computer experiments using Gaussian processes [45]. Finally, the models upon which this calibration procedure have been applied are very simplistic. We plan in the future to apply this to the robust calibration of the bottom friction of a realistic model of the ocean.

\section{References}

[1] W. E. Walker, P. Harremoës, J. Rotmans, J. P. van der Sluijs, M. B. van Asselt, P. Janssen, M. P. Krayer von Krauss, Defining uncertainty: A conceptual basis for uncertainty management in model-based decision support, Integrated assessment 4 (2003) 5-17.

[2] S. K. Das, R. W. Lardner, On the estimation of parameters of hydraulic models by assimilation of periodic tidal data, Journal of Geophysical Research 96 (1991) 15187.

[3] S. K. Das, R. W. Lardner, Variational parameter estimation for a two-dimensional numerical tidal model, International Journal for Numerical Methods in Fluids 15 (1992) 313-327.

[4] M. Boutet, Estimation Du Frottement Sur Le Fond Pour La Modélisation de La Marée Barotrope, Ph.D. thesis, Université d'Aix Marseille, 2015. 
[5] P. G. J. ten Brummelhuis, A. W. Heemink, Parameter identification in tidal models with uncertain boundary conditions, Stochastic Hydrology and Hydraulics 4 (1990) 193-208.

[6] L. Huyse, D. M. Bushnell, Free-form airfoil shape optimization under uncertainty using maximum expected value and second-order second-moment strategies (2001).

[7] G. Kuczera, B. Renard, M. Thyer, D. Kavetski, There are no hydrological monsters, just models and observations with large uncertainties!, Hydrological Sciences Journal 55 (2010) 980-991.

[8] T. E. Wong, A. Klufas, V. Srikrishnan, K. Keller, Neglecting model structural uncertainty underestimates upper tails of flood hazard, Environmental Research Letters 13 (2018) 074019.

[9] Y. Hu, S. Rao, Robust Design of Horizontal Axis Wind Turbines Using Taguchi Method, Journal of Mechanical Design 133 (2011) 111009.

[10] L. Brevault, Contributions to Multidisciplinary Design Optimization under Uncertainty, Application to Launch Vehicle Design., Theses, Ecole Nationale Supérieure des Mines de Saint-Étienne, 2015.

[11] N. Lelièvre, P. Beaurepaire, C. Mattrand, N. Gayton, A. Otsmane, On the consideration of uncertainty in design: Optimization-reliability-robustness, Structural and Multidisciplinary Optimization 54 (2016) 1423-1437.

[12] G. Petrone, G. Iaccarino, D. Quagliarella, Robustness criteria in optimization under uncertainty, Evolutionary and deterministic methods for design, optimization and control (EUROGEN 2011). CIRA, Capua (2011) 244-252.

[13] P. Seshadri, P. Constantine, G. Iaccarino, G. Parks, A density-matching approach for optimization under uncertainty, arXiv:1409.7089 [math, stat] (2014).

[14] L. W. Cook, J. P. Jarrett, Horsetail matching: A flexible approach to optimization under uncertainty, Engineering Optimization 50 (2018) 549-567.

[15] C. Ning, F. You, Optimization under uncertainty in the era of big data and deep learning: When machine learning meets mathematical programming, Computers \& Chemical Engineering 125 (2019) 434-448.

[16] P. J. Huber, Robust statistics, in: International Encyclopedia of Statistical Science, Springer, 2011, pp. 1248-1251.

[17] V. Rao, A. Sandu, M. Ng, E. Nino-Ruiz, Robust data assimilation using $\$ L_{-} 1 \$$ and Huber norms, SciRate (2015).

[18] J. O. Berger, E. Moreno, L. R. Pericchi, M. J. Bayarri, J. M. Bernardo, J. A. Cano, J. De la Horra, J. Martín, D. Ríos-Insúa, B. Betrò, An overview of robust Bayesian analysis, Test 3 (1994) 5-124.

[19] H. Rahimian, S. Mehrotra, Distributionally Robust Optimization: A Review, arXiv:1908.05659 [cs, math, stat] (2019).

[20] J. Marzat, E. Walter, H. Piet-Lahanier, Worst-case global optimization of black-box functions through Kriging and relaxation, Journal of Global Optimization 55 (2013) 707-727.

[21] J. Villemonteix, E. Vazquez, E. Walter, An informational approach to the global optimization of expensive-to-evaluate functions, arXiv:cs/0611143 (2006).

[22] P. Hennig, C. J. Schuler, Entropy Search for Information-Efficient Global Optimization (2011).

[23] J. M. Buhmann, M. Mihalak, R. Sramek, P. Widmayer, Robust optimization in the presence of 
uncertainty, in: Proceedings of the 4th Conference on Innovations in Theoretical Computer Science - ITCS '13, ACM Press, Berkeley, California, USA, 2013, p. 505. doi:10.1145/2422436.2422491.

[24] P. Kouvelis, A. A. Kurawarwala, G. J. Gutiérrez, Algorithms for robust single and multiple period layout planning for manufacturing systems, European Journal of Operational Research 63 (1992) $287-303$.

[25] L. Snyder, M. Daskin, Stochastic p-robust location problems, Iie Transactions 38 (2004).

[26] A. Juditsky, A. S. Nemirovski, G. Lan, A. Shapiro, Stochastic Approximation Approach to Stochastic Programming, in: ISMP 2009 - 20th International Symposium of Mathematical Programming, 2009.

[27] S. Kim, R. Pasupathy, S. Henderson, A Guide to Sample Average Approximation, volume 216, 2015, pp. 207-243. doi:10.1007/978-1-4939-1384-8-8.

[28] J. Janusevskis, R. Le Riche, Simultaneous Kriging-Based Sampling for Optimization and Uncertainty Propagation, Technical Report, 2010.

[29] V. Baudoui, Optimisation Robuste Multiobjectifs Par Modèles de Substitution, Ph.D. thesis, Toulouse, ISAE, 2012.

[30] O. Grodzevich, O. Romanko, Normalization and other topics in multi-objective optimization (2006).

[31] R. T. Marler, J. S. Arora, The weighted sum method for multi-objective optimization: New insights, Structural and Multidisciplinary Optimization 41 (2010) 853-862.

[32] J. S. Lehman, T. J. Santner, W. I. Notz, Designing computer experiments to determine robust control variables, Statistica Sinica (2004) 571-590.

[33] B. Silverman, Density Estimation: For Statistics and Data Analysis, 2018. doi:10.1201/ 9781315140919.

[34] A. P. Dempster, N. M. Laird, D. B. Rubin, Maximum likelihood from incomplete data via the EM algorithm, Journal of the royal statistical society. Series B (methodological) (1977) 1-38.

[35] D. Freedman, P. Diaconis, On the histogram as a density estimator:L2 theory, Zeitschrift für Wahrscheinlichkeitstheorie und Verwandte Gebiete 57 (1981) 453-476.

[36] D. W. Scott, On Optimal and Data-Based Histograms, Biometrika 66 (1979) 605.

[37] R. T. Rockafellar, S. P. Uryasev, M. Zabarankin, Deviation Measures in Risk Analysis and Optimization, SSRN Scholarly Paper ID 365640, Social Science Research Network, Rochester, NY, 2002 .

[38] L. J. Savage, The Theory of Statistical Decision, Journal of the American Statistical Association 46 (1951) 55-67.

[39] J. Luedtke, S. Ahmed, A sample approximation approach for optimization with probabilistic constraints, SIAM Journal on Optimization 19 (2008) 674-699.

[40] M. Thulin, The cost of using exact confidence intervals for a binomial proportion, Electronic Journal of Statistics 8 (2014) 817-840.

[41] M. C. Kennedy, A. O'Hagan, Bayesian calibration of computer models, Journal of the Royal Statistical Society: Series B (Statistical Methodology) 63 (2001) 425-464.

[42] D. Ginsbourger, J. Baccou, C. Chevalier, F. Perales, N. Garland, Y. Monerie, Bayesian Adaptive 
Reconstruction of Profile Optima and Optimizers, SIAM/ASA Journal on Uncertainty Quantification 2 (2014) 490-510.

[43] J. Bossek, B. Bischl, T. Wagner, G. Rudolph, Learning Feature-Parameter Mappings for Parameter Tuning via the Profile Expected Improvement, in: Proceedings of the 2015 Annual Conference on Genetic and Evolutionary Computation, GECCO '15, ACM, New York, NY, USA, 2015, pp. 13191326. doi:10.1145/2739480.2754673.

[44] T. Gneiting, M. Katzfuss, Probabilistic Forecasting, Annual Review of Statistics and Its Application 1 (2014) 125-151.

[45] D. Ginsbourger, R. Le Riche, Towards Gaussian Process-based Optimization with Finite Time Horizon, in: A. Giovagnoli, A. C. Atkinson, B. Torsney, C. May (Eds.), mODa 9 - Advances in Model-Oriented Design and Analysis, Contributions to Statistics, Physica-Verlag HD, Heidelberg, 2010, pp. 89-96. doi:10.1007/978-3-7908-2410-0_12. 